\title{
Identification of Cuticular and Web Lipids of the Spider Argiope bruennichi
}

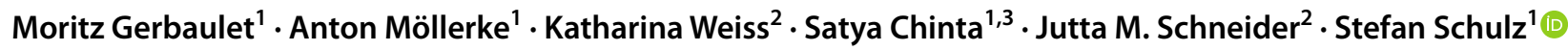

Received: 23 August 2021 / Revised: 18 November 2021 / Accepted: 22 November 2021 / Published online: 10 January 2022

(c) The Author(s) 2022

\begin{abstract}
Emerging evidence shows that the cuticular and silk lipids of spiders are structurally more diverse than those of insects, although only a relatively low number of species have been investigated so far. As in insects, such lipids might play a role as signals in various contexts. The wasp spider Argiope bruennichi has probably the best investigated chemical communication system within spiders, including the known structure of the female sex pheromone. Recently we showed that kin-recognition in A. bruennichi could be mediated through the cuticular compounds consisting of hydrocarbons and, to a much larger proportion, of wax esters. By use of mass spectrometry and various derivatization methods, these were identified as esters of 2,4-dimethylalkanoic acids and 1-alkanols of varying chain lengths, such as tetradecyl 2,4-dimethylheptadecanoate. A representative enantioselective synthesis of this compound was performed which proved the identifications and allowed us to postulate that the natural enantiomer likely has the $(2 R, 4 R)$-configuration. Chemical profiles of the silk and cuticular lipids of females were similar, while male cuticular profiles differed from those of females. Major components of the male cuticular lipids were tridecyl 2,4-dimethyl- $\mathrm{C}_{17-19}$ alkanoates, whereas those of females were slightly longer, comprising tridecyl 2,4-dimethyl- $\mathrm{C}_{19-21}$ alkanoates. In addition, minor female-specific 4-methylalkyl esters were detected.
\end{abstract}

Keywords GC/MS $\cdot$ Wax esters $\cdot$ Branched fatty acids $\cdot$ Pheromones $\cdot$ Kin-recognition

\section{Introduction}

Chemical communication is the predominant form of communication in arthropods because their typically small size limits the efficacy of, for example, visual or acoustic communication (Greenfield 2002). Often, cuticular lipids, besides their role as a water barrier (Gibbs and Rajpurohit 2010), have important communicative functions, as is well documented for insects (Howard and Blomquist 2005,

In Memory of Prof. Dr. Dr. hc mult. Wittko Francke. May he rest in peace.

Stefan Schulz

stefan.schulz@tu-bs.de

1 Institute of Organic Chemistry, TU Braunschweig, Hagenring 30, 38106 Braunschweig, Germany

2 Institute of Zoology, Universität Hamburg, Martin-Luther-King Platz 3, 20146 Hamburg, Germany

3 U.S. Department of Agriculture, Agricultural Research Service, 1600-1700 SW 23rd Drive, Gainesville, FL 32608, USA
Blomquist and Bagnères 2010). In arthropods other than insects, however, cuticular chemistry and its role in communication is largely unknown.

For example, in spiders, the chemistry of cuticular lipids and especially silk has been investigated in only a few species. Generally, the composition of cuticular lipids seems to be more divers in spiders than in insects. In a couple of previous studies it was shown that after alkanes, the dominating compound class within insects (Blomquist and Bagnères 2010), major components can be long-chain, methylbranched methyl ethers. These have been reported from linyphiids (Schulz and Toft 1993), Nephila clavipes (Schulz 2001), and Tetragnatha sp. (Adams et al. 2021). Fatty acids and aliphatic saturated alcohols occur in various other species (Trabalon et al. 1996, 1997; Prouvost et al. 1999; Trabalon and Assi-Bessekon 2008; Trabalon 2011). These compounds can be combined to form esters. Such esters can be simple methyl esters of fatty acids (Prouvost et al. 1999; Trabalon 2011), but some species have evolved more elaborate compounds. The social spider Anelosimus eximus produces a complex array of long-chain methyl branched $n$-propyl esters, e. g. propyl 4,20-dimethylhentriacontanoate 
(Bagnères et al. 1997). Another ester type is present on the cuticle of the spider Argyrodes elevatus (Theridiidae), consisting of short, partly branched alcohols conjugated to longer acids, occurring in completely different sex-specific mixtures of a few compounds. These include undecyl 2-methyltridecanoate in males and 2,8-dimethylundecyl 2,8-dimethylundecanoate as well as heptadecyl 4-methylheptanoate in females. Other spiders exclusively use insect-type hydrocarbons (Trabalon and Assi-Bessekon 2008; Grinsted et al. 2011). As in insects, these cuticular constituents were suggested to transmit intraspecific information in various ways (Witte et al. 2009; Trabalon and Bagnères 2010; Xiao et al. 2010; Grinsted et al. 2011; Beeren et al. 2012; Schulz 2013; Ruhland et al. 2019; Adams et al. 2021), although compared to volatile pheromones in spiders (Schulz 2004, 2013; Gaskett 2007; Fischer 2019) this area is less well studied.

Among spiders, the chemical communication system of the European wasp spider Argiope bruennichi is probably one of the best-studied. As entelegyne spiders, genital structures are paired in both sexes, but only one can be used during a copulation (Foelix 2011). After sperm transfer, males effectively plug the genital opening of the female with parts of their genitalia that are rendered dysfunctional thereafter (Nessler et al. 2007; Uhl et al. 2010). Most males fall victim to sexual cannibalism after their first copulation, leaving one genital opening of the female available for another male. Males that escape and copulate twice can monopolize paternity with a female because both openings are securely plugged. Consequently, A. bruennichi females and males are restricted to a maximum of two copulations. Females are known to produce a volatile pheromone, trimethyl methylcitrate, that attracts males in the field and elicits courtship behavior (Chinta et al. 2010). Behavioral experiments indicate, however, that males also use the pheromone to assess a female's condition, age, and mating status. For example, field observations (Welke et al. 2012) and laboratory experiments (Cory and Schneider 2016) demonstrated that males are more likely to monopolize females that are relatively heavy and old suggesting that females modulate pheromone release according to their state (Cory and Schneider 2016). Furthermore, mating experiments with siblings and non-siblings indicate that males are also capable of kin recognition. While male A. bruennichi readily copulate with sisters in laboratory mating trials, they terminate copulation earlier as compared to unrelated females (Welke and Schneider 2010). Consequently, they survive copulation more often (Welke and Schneider 2010), allowing them to leave and search for another female. Recently some of us showed that kin-recognition might be mediated by cuticular compounds occurring in family-specific bouquets and suggested an important role of unusual long-chain esters in kin recognition (Weiss and Schneider 2021). We present here the identification and synthesis of the dominant group of compounds of these bouquets, long chain esters of 2,4-dimethylbranched acids with mostly unbranched fatty alcohols, and clarify their distribution.

\section{Methods and Materials}

\section{Spider Extracts}

Subadult male and female Argiope bruennichi (Scopoli, 1772) (Araneae, Araneidae) were collected from natural meadows in Northern Germany (Buxtehude, Harmstorf, Pevestorf, Lower Saxony; Wedel, Schleswig-Holstein), between 24 June and 5 July 2019. Argiope bruennichi is common throughout Europe and its collection requires no permits. Spiders were transferred to the laboratory at the University of Hamburg, Germany, where they were individually housed in upturned plastic cups $(250$ or $500 \mathrm{~mL}$ depending on the spider's size) with a hole in the bottom stuffed with cotton wool. Spiders were kept under natural light conditions at a constant temperature of $25^{\circ} \mathrm{C}$ and relative humidity of $45 \%$. Spiders were checked for adult molts daily. In both sexes, subadult and adult spiders can be unambiguously distinguished by their genitalia (Uhl et al. 2007). A spider's age is defined here as days since adult molt. Twice a week, subadult spiders were provided with approximately 15 Drosophila spp. and adult females with three Calliphora sp. houseflies. Adult males were fed with approximately 10 Drosophila spp. once a week. All spiders were provided with water from a sprayer at least six days a week. Twenty virgin male (mean age \pm SD: $13.5 \pm 1.2$ days) and 30 virgin female A. bruennichi (mean age \pm SD: $11.8 \pm 0.9$ days) were used for chemical analysis.

\section{Analysis by coupled gas chromatography-mass spectrometry (GC/MS)}

To obtain silk samples, the females were placed into clean Perspex frames $(35 \times 35 \times 6 \mathrm{~cm})$ and allowed to build a web. After $24 \mathrm{~h}$, spiders were removed from their webs and the silk was collected by slowly winding it around a glass Pasteur-pipet washed with ethanol. The tip of the pipet holding the silk was then snapped off into a small glass vial. Males and females were placed in clean glass vials and cold anesthetized. All samples were stored at $-25^{\circ} \mathrm{C}$ until analysis. Cuticular extracts were prepared by placing individual females in $3 \mathrm{~mL}$ of dichloromethane (DCM; GC/MS grade, Merck, Darmstadt, Germany) and the smaller males in $1 \mathrm{~mL}$ DCM for one hr. Silk samples were extracted in $1 \mathrm{~mL} \mathrm{DCM}$. Extracts were concentrated by evaporation at room temperature to approximately $90 \mu \mathrm{l}$ (females) and $50 \mu \mathrm{l}$ (males and silk), respectively. An aliquot of $1 \mu \mathrm{l}$ of each sample was 
analyzed by GC/MS on a Shimadzu GCMS-QP2010S system (Shimadzu Corporation, Kyoto, Japan). After individual analyses, the female silk and body extracts and male samples were pooled separately for further chemical identification. For the analysis of samples of individuals, the gas chromatograph was equipped with an SH-Rtx-5MS fused silica capillary column $(30 \times 0.25 \mathrm{~mm}$ ID, $0.25 \mu \mathrm{m}$ film thickness; Shimadzu Corporation, Kyoto, Japan). The oven temperature was programmed from 80 to $260{ }^{\circ} \mathrm{C}$ at $30^{\circ} / \mathrm{min}$ and from 260 to $300{ }^{\circ} \mathrm{C}$ at $1 \% \mathrm{~min}$, with a 1 -min initial isothermal and a 10-min final isothermal hold. A split-splitless injector was used at $250{ }^{\circ} \mathrm{C}$ in splitless mode. The carrier gas was helium at a constant flow rate of $1 \mathrm{~mL} / \mathrm{min}$. The ionization voltage of the electron ionization mass spectrometer was $70 \mathrm{eV}$. Source temperature was $200^{\circ} \mathrm{C}$ and the interface temperature was $280^{\circ} \mathrm{C}$. Data acquisition and storage were performed with the software GCMSsolution (Version 4.45; Shimadzu Corporation, Kyoto, Japan). Peak areas were obtained by manual integration using the GCMSsolution software. Linear retention indices of all substances were calculated according to van den Dool and Kratz (1963). $n$-Alkanes were identified by comparing their mass spectra with those of authentic reference compounds. Alkenes were identified by their typical mass spectra. Methyl-branched hydrocarbons were identified by diagnostic ions resulting from their typical $\alpha$-cleavage at the position of the methyl branch and by a fragment at M-15 if the molecular ion was not detected. Moreover, their linear retention indices were compared to published values (Carlson et al. 1998; El-Sayed 2019) and also to calculated theoretical values as described by Schulz (2001). Other compounds were tentatively identified by comparing their mass spectra and linear retention indices with those of a database (NIST 08 mass spectral library 2008). Mean relative peak areas were calculated by standardizing total mean peak areas to $100 \%$. GC/MS analyses of pooled samples were performed on a GC 7890A coupled to a 5975C mass selective detector (MSD, Agilent Technologies, Germany). The gas chromatograph was equipped with an HP-5 MS column $(30 \times 0.25 \mathrm{~mm}$ ID, $0.25 \mu \mathrm{m}$ film thickness; Agilent Technologies, Inc. USA) with helium as the carrier gas. The combined samples were analyzed with a temperature program of $50{ }^{\circ} \mathrm{C}$ for $5 \mathrm{~min}$ that increased by $3{ }^{\circ} \mathrm{C} / \mathrm{min}$ to $320^{\circ} \mathrm{C}$ with a final hold time of $10 \mathrm{~min}$. Derivatized samples were analyzed with a temperature program of $50{ }^{\circ} \mathrm{C}$ for $5 \mathrm{~min}$, increased by $5{ }^{\circ} \mathrm{C} / \mathrm{min}$ to $320{ }^{\circ} \mathrm{C}$ with a final hold time of $10 \mathrm{~min}$. To compare the chemical composition between samples, relative peak areas were calculated for each of the 20 male, 30 female and web silk extracts separately by standardizing the total peak area of each extract to $100 \%$. Values given in Table 1 are means \pm standard deviation (SD). GC on chiral phases was performed using BetaDex $^{\text {TM }} 225$ (Sigma, $30.0 \mathrm{~m} \times 0.25 \mathrm{~mm}$ ) or Hydrodex $\beta$-6TBDM (Machery and Nagel, $30.0 \mathrm{~m} \times 0.25 \mathrm{~mm}$ ) columns with a flow of $1.5 \mathrm{~mL} \mathrm{~min}{ }^{-1}$ hydrogen as the carrier gas and a flame ionization detector. Individual time programs are given in the respective experimental descriptions and Figs. 7 and S5.

\section{Discriminant analyses}

Individual chemical profiles of 20 male and 29 female cuticles, as well as 22 female webs were compared by discriminant analyses (DA) in SPSS 27.0. One female and eight silk extracts were excluded from the analyses, as only a few peaks were detected in their GC profiles. Only peaks representing $>1 \%$ of the mean total peak area in all three sample types were considered (seven peaks in total: $I 2700,2900$, 3061, 3273, 3380, 3476, 3575 in Table 1). Because relative peak areas constitute compositional data, they were transformed to log-contrasts before statistical analyses (Aitchison 2003). Separate DAs were conducted using all seven peaks $>1 \%$, only wax esters (four peaks), and only hydrocarbons (three peaks), respectively. Relative areas of peaks entering a given DA were again calculated by standardizing their total peak area to $100 \%$.

\section{Microreactions of Extracts}

The extracts were derivatized in microreactions to obtain more structural information about methyl-branch positions of the acid and alcohol components of the long chain esters. Extracts were transesterified with trimethylsulfonium hydroxide (TMSH) (Müller et al. 1990) to form the corresponding methyl esters and free alcohols (Fig. 1). Subsequent transesterification of the methyl esters with sodium 3-pyridinylmethoxide to form the corresponding 3-pyridinylmethyl esters was performed (Harvey 1982) as well as esterification of the free alcohols with nicotinic acid to form the corresponding esters (Vetter and Meister 1981; Harvey 1991).

\section{Transesterification with Trimethylsulfonium Hydroxide}

Trimethylsulfonium hydroxide (TMSH, $100 \mu \mathrm{l}, 0.25 \mathrm{~m}$ in methanol) was added to the natural sample (in $20 \mu \mathrm{l}$ dichloromethane) in a GC-vial $(2 \mathrm{~mL})$. The reaction mixture was placed in a heating block at $90{ }^{\circ} \mathrm{C}$ for $6 \mathrm{~h}$ and regularly shaken vigorously. The solvents and reagents were removed with a stream of nitrogen and the residue was dissolved in DCM (20 $\mu$ l) (Müller et al. 1990).

\section{Transesterification with 3-Pyridinylmethanol}

3-Pyridinylmethanol $(50 \mu \mathrm{l})$ was added to freshly cut sodium $(0.2 \mathrm{mg})$ in a GC-vial $(2 \mathrm{~mL})$. The reaction mixture was heated to $80{ }^{\circ} \mathrm{C}$ in a heating block until the sodium had 
Fig. 1 Microreactions performed with $A$. bruennichi extracts. Transesterification with TMSH transformed the wax esters into methyl esters that were again transesterified with 3-pyridinylmethanol or transformed into nicotinates with nicotinic acid<smiles>[R]OC([R])=O</smiles>

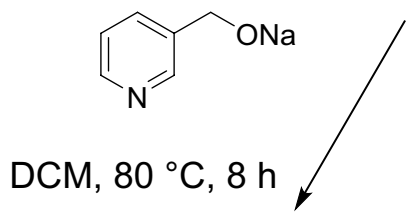<smiles>O=C(O)c1cccnc1</smiles><smiles>[R]C(=O)OCc1cccnc1</smiles><smiles>[R]OC(=O)c1cccnc1</smiles>

dissolved. Sodium 3-pyridinylmethoxide (2\% in 3-pyridinylmethanol) was obtained as a syrupy yellow liquid. The freshly prepared sodium 3-pyridinylmethoxide (2 drops, $2 \%$ in 3-pyridinylmethanol) was added to the methyl ester sample $(20 \mu \mathrm{l}$ in dichloromethane) in a GC-vial $(2 \mathrm{~mL})$. The reaction was placed in a heating block at $80^{\circ} \mathrm{C}$ for $3 \mathrm{~h}$ and regularly shaken vigorously. Methanol $(200 \mu \mathrm{l})$ and water ( 3 drops) were added to the solution and the mixture was extracted with pentane $(3 \times 200 \mu \mathrm{l})$. The pentane phases were combined, the solvent was removed under a stream of nitrogen, and the residue was dissolved in DCM $(20 \mu \mathrm{l})$.

\section{Preparation of Nicotinates}

DCM $(50 \mu \mathrm{l})$, nicotinic acid (1 mg), 1-ethyl-3-(3-dimethylaminopropyl)carbodiimide (EDC, $1 \mathrm{mg}$ ) and 4-dimethylaminopyridine (DMAP, catalytic amount) were added to the sample of the methyl esters and free alcohols $(50 \mu \mathrm{l}$ in dichloromethane) in a GC-vial $(2 \mathrm{~mL})$. The reaction mixture was kept at room temperature for $2 \mathrm{~h}$ and regularly shaken vigorously. The solvents were removed in a stream of nitrogen and the residue was extracted with pentane $(3 \times 100 \mu \mathrm{l})$. The combined pentane phases were again concentrated in a stream of nitrogen and the residue was dissolved in DCM $(50 \mu \mathrm{l})$.

\section{General Experimental Procedures}

All reactions were performed in oven-dried glassware under a nitrogen atmosphere. Solvents were dried according to standard procedures. Column chromatography was done with silica $60(0.063-0.200 \mathrm{~mm}, 70-230$ mesh ASTM) and thin layer chromatography (TLC) with Polygram ${ }^{\circledR}$ SIL G/UV silica 60 plates, $0.20 \mathrm{~mm}$ thickness. Compounds were visualized with potassium permanganate solution. NMR spectra were recorded either on Avance III HD $300 \mathrm{~N}\left({ }^{1} \mathrm{H}-\mathrm{NMR}: 300 \mathrm{MHz},{ }^{13} \mathrm{C}-\mathrm{NMR}: 76 \mathrm{MHz}\right)$, DRX $400\left({ }^{1} \mathrm{H}-\mathrm{NMR}: 400 \mathrm{MHz},{ }^{13} \mathrm{C}-\mathrm{NMR}: 101 \mathrm{MHz}\right)$, AVII 400 ( ${ }^{1} \mathrm{H}-\mathrm{NMR}: 400 \mathrm{MHz},{ }^{13} \mathrm{C}-\mathrm{NMR}$ : $101 \mathrm{MHz}$ ), or AVII 600 $\left({ }^{1} \mathrm{H}-\mathrm{NMR}\right.$ : $\left.600 \mathrm{MHz}\right)$ instruments. Data are reported as follows: chemical shifts, multiplicity $(\mathrm{s}=$ singlet, $\mathrm{d}=$ doublet, $\mathrm{t}=$ triplet, $\mathrm{q}=$ quartet, $\mathrm{m}=$ multiplet), coupling constants $(\mathrm{Hz})$. IR spectra were measured on a Bruker Tensor 27 (diamond-ATR). Mass spectra were recorded with a combination of an Agilent Technologies 5977B gas chromatograph connected to an Agilent Technologies 8860 Series MSD. The enantiomeric excess of chiral compounds was determined by GC on BetaDex ${ }^{\text {TM }} 225$ or Hydrodex $\beta-6$ TBDM [ $(30.0 \mathrm{~m} \times 0.25 \mathrm{~mm})$ phases, operated with $\mathrm{H}_{2}$ as carrier gas with a flow of $1.5 \mathrm{ml} \mathrm{min}$. Optical rotations were determined with an MCP 150 polarimeter (Anton Paar) with a cell length of $10 \mathrm{~cm}$ ( $c$ given in $\mathrm{mg} / \mathrm{mL}$ ).

\section{Preparation of S-Ethyl (S)-4-((tert-Butyldiphenylsilyl) oxy)-3-methylbutanethioate (2)}

The synthesis was performed according to Horst et al. (2007) using $(R)-1-\left[\left(S_{\mathrm{P}}\right)\right.$-2-(diphenylphosphino)ferrocenyl]ethyldicyclohexylphosphine (Josiphos, 11, $0.08 \mathrm{mmol}$, $50 \mathrm{mg}, 0.012$ eq.) and $\mathrm{CuBr} \cdot \mathrm{SMe}_{2}(0.065 \mathrm{mmol}, 13 \mathrm{mg}$, 0.01 eq.), methyl tert-butyl ether (MTBE, $50 \mathrm{~mL}$ ), $\mathrm{MeMgBr}$ (7.78 mmol, solution in diethyl ether), and $S$-ethyl $(E)$ 4-((tert-butyldiphenylsilyl)oxy)but-2-enethioate $(6.48 \mathrm{mmol}$, $2493 \mathrm{mg}, 1 \mathrm{eq}$.) to afford $\mathbf{2}$ as a colorless oil (2199 mg, 85\%). $[\alpha]_{\mathrm{D}}^{20}=-4.60\left(10 \mathrm{mg} / \mathrm{mL} ; \mathrm{CH}_{2} \mathrm{Cl}_{2}\right)$. FT-IR: $\nu / \mathrm{cm}^{-1}=2960$, 2895, 2859, 1688, 1466, 1427, 1389, 1261, 1150, 1108, 1040, 1005, 941, 821, 802, 763, 741, 703, 615. ${ }^{1} \mathrm{H}-\mathrm{NMR}$ : $\left(400 \mathrm{MHz}, \mathrm{CDCl}_{3}\right) \delta / \mathrm{ppm}=7.71-7.66(\mathrm{~m}, 4 \mathrm{H}), 7.47-7.37$ (m, 6H), 3.53 (ddd, $J=16.2,9.9,5.7 \mathrm{~Hz}, 2 \mathrm{H}$ ), 2.93-2.82 (m, 3H), 2.40 (dd, $J=14.4,8.4 \mathrm{~Hz}, 1 \mathrm{H}), 2.36-2.25$ (m, 1H), 
$1.26(\mathrm{t}, J=7.4 \mathrm{~Hz}, 3 \mathrm{H}), 1.08(\mathrm{~d}, J=2.8 \mathrm{~Hz}, 9 \mathrm{H}), 0.98(\mathrm{~d}$, $J=6.6 \mathrm{~Hz}, 3 \mathrm{H}) .{ }^{13} \mathrm{C}-\mathrm{NMR}$, DEPT: $\left(101 \mathrm{MHz}, \mathrm{CDCl}_{3}\right) \delta$ / ppm $=199.2(\mathrm{C}=\mathrm{O}), 135.7\left(\mathrm{CH}_{\mathrm{Ar}}\right), 135.7\left(\mathrm{CH}_{\mathrm{Ar}}\right), 133.8$ $\left(\mathrm{CH}_{\mathrm{Ar}}\right), 133.8\left(\mathrm{C}_{\mathrm{Ar}}\right), 129.7\left(\mathrm{CH}_{\mathrm{Ar}}\right), 127.7\left(\mathrm{CH}_{\mathrm{Ar}}\right), 68.0$ $\left(\mathrm{CH}_{2}\right), 47.7\left(\mathrm{CH}_{2}\right), 33.9(\mathrm{CH}), 27.0\left(\mathrm{CH}_{3}\right), 23.4\left(\mathrm{CH}_{2}\right), 19.4$ (C), $16.6\left(\mathrm{CH}_{3}\right), 14.9\left(\mathrm{CH}_{3}\right)$. EI-MS $(70 \mathrm{eV}): \mathrm{m} / \mathrm{z}=344(23)$, $343\left(\left[M-{ }^{t} \mathrm{Bu}\right]^{+}, 76\right), 244$ (26), 243 (100), 197 (13), 183 (39), 181 (22), 135 (25), 137 (23), 105 (16). The enantiomeric excess (ee) was determined by cleavage of the silyl ether using tetrabutylammonium fluoride (TBAF) (Horst et al. 2007). The resulting lactone was analyzed by GC on a chiral phase BetaDex ${ }^{\mathrm{TM}} 225(30.0 \mathrm{~m} \times 0.25 \mathrm{~mm}$, initial temp. $50{ }^{\circ} \mathrm{C}$ then $10{ }^{\circ} \mathrm{C} \mathrm{min}{ }^{-1}$ to final temp. $160{ }^{\circ} \mathrm{C}$, Fig. S5). Retention time, 13.75 min (minor), 13.85 (major) showed 94\% ee (Lit.: $98 \%$ ee Horst et al. 2007).

\section{Preparation of S-Ethyl (2E,5S,2E)-6-((tert-Butyldiphenylsilyl) oxy)-5-methylhex-2-enethioate (3)}

The synthesis was performed as described by Horst et al. (2007) using $\mathrm{Et}_{3} \mathrm{SiH}$ (36.69 mmol, $4266 \mathrm{mg}, 3$ eq.), 2 (12.23 mmol, $4900 \mathrm{mg}, 1$ eq.) and 10\% $\mathrm{Pd} / \mathrm{C}(5 \mathrm{~mol} \%$, $650 \mathrm{mg})$ in $\mathrm{CH}_{2} \mathrm{Cl}_{2}(20 \mathrm{~mL})$ to afford the crude aldehyde that underwent a Wittig reaction with $S$-ethyl 2-(triphenyl- $\lambda^{5}$ phosphaneylidene)ethanethioate $(3.221 \mathrm{mmol}, 1174 \mathrm{mg})$ in $\mathrm{CH}_{2} \mathrm{Cl}_{2}(40 \mathrm{~mL})$ to afford 3 as a colorless oil $(2010 \mathrm{mg}, 39 \%$ over two steps). $[\alpha]_{\mathrm{D}}^{20}=-6.2\left(10 \mathrm{mg} / \mathrm{mL} ; \mathrm{CHCl}_{3}\right) .{ }^{1} \mathrm{H}-\mathrm{NMR}$ : $\left(300 \mathrm{MHz}, \mathrm{CDCl}_{3}\right) \delta / \mathrm{ppm}=7.70-7.60(\mathrm{~m}, 4 \mathrm{H}), 7.46-7.33$ (m, 6H), 6.93-6.79 (m, 1H), $6.11(\mathrm{~d}, J=15.5 \mathrm{~Hz}, 1 \mathrm{H}), 3.49$ (ddd, $J=16.3,10.0,5.9 \mathrm{~Hz}, 2 \mathrm{H}), 2.94$ (q, $J=7.4 \mathrm{~Hz}, 2 \mathrm{H}$ ), 2.50-2.36 (m, 1H), 2.04 (ddd, $J=15.4,8.4,7.3 \mathrm{~Hz}, 1 \mathrm{H}$ ), 1.94-1.77 (m, 1H), $1.28(\mathrm{dd}, J=7.7,7.2 \mathrm{~Hz}, 3 \mathrm{H}), 1.06(\mathrm{~s}$, 9H), 0.91 (d, $J=6.8 \mathrm{~Hz}, 3 \mathrm{H}) .{ }^{13} \mathrm{C}-\mathrm{NMR}$, DEPT: $(76 \mathrm{MHz}$, $\left.\mathrm{CDCl}_{3}\right) \delta / \mathrm{ppm}=190.1(\mathrm{C}), 144.0(\mathrm{CH}), 135.7(\mathrm{CH}), 135.7$ $(\mathrm{CH}), 133.9(\mathrm{CH}), 133.8(\mathrm{CH}), 130.1,129.8(\mathrm{CH}), 127.8$ $(\mathrm{CH}), 68.2\left(\mathrm{CH}_{2}\right), 36.1\left(\mathrm{CH}_{2}\right), 35.6(\mathrm{CH}), 27.0\left(\mathrm{CH}_{3}\right), 23.2$ $\left(\mathrm{CH}_{2}\right), 19.4(\mathrm{C}), 16.6\left(\mathrm{CH}_{3}\right), 15.0\left(\mathrm{CH}_{3}\right)$. EI-MS $(70 \mathrm{eV})$ : $m / z=283\left(\left[M-{ }^{t} \mathrm{Bu}\right]^{+}, 31\right), 200(19), 199$ (100), 181 (24), 175 (16), 139 (38), 105 (18), 83 (23), 77 (19), 41 (16).

\section{Preparation of S-Ethyl (3R,5S)-6-((tert-Butyldiphenylsilyl) oxy)-3,5-dimethylhexanethioate (4)}

The synthesis was performed according to HoRst et al. (2007) using 11 ( $0.12 \mathrm{mmol}, 75 \mathrm{mg}, 0.012$ eq.), $\mathrm{CuBr} \cdot \mathrm{SMe}_{2}$ (0.097 mmol, $20 \mathrm{mg}, 0.01$ eq.) dissolved in MTBE $(65 \mathrm{~mL})$, $\mathrm{MeMgBr}$ (11.64 mmol, solution in diethyl ether), and $\mathbf{3}$ (9.70 mmol, $3730 \mathrm{mg}, 1$ eq.) to afford 4 as a colorless oil (3533 mg, 82\%). $[\alpha]_{\mathrm{D}}^{20}=-4.3\left(10 \mathrm{mg} / \mathrm{mL} ; \mathrm{CHCl}_{3}\right)$. FT-IR: $\nu / \mathrm{cm}^{-1}=2959,2930,2860,1689,1463,1427,1384,1262$, 1108, 1083, 1002, 821, 741, 702, 615. ${ }^{1} \mathrm{H}-\mathrm{NMR}$ : (300 MHz, $\left.\mathrm{CDCl}_{3}\right) \delta / \mathrm{ppm}=7.72-7.66(\mathrm{~m}, 4 \mathrm{H}), 7.47-7.36(\mathrm{~m}, 6 \mathrm{H})$,
3.48 (ddd, $J=24.5,9.8,5.9 \mathrm{~Hz}, 2 \mathrm{H}), 2.88$ (q, $J=7.5 \mathrm{~Hz}, 2 \mathrm{H}$ ), $2.53(\mathrm{dd}, J=14.3,5.0 \mathrm{~Hz}, 1 \mathrm{H}), 2.26(\mathrm{dd}, J=14.3,8.7 \mathrm{~Hz}$, $1 \mathrm{H}), 2.18-2.02(\mathrm{~m}, 1 \mathrm{H}), 1.81-1.65(\mathrm{~m}, 1 \mathrm{H}), 1.48-1.35(\mathrm{~m}$, 1H), 1.30-1.21 (m, 3H), 1.12-1.05 (m, 9H), 1.05-0.98 $(\mathrm{m}, 1 \mathrm{H}), 0.93(\mathrm{dt}, J=6.7,4.5 \mathrm{~Hz}, 6 \mathrm{H}) .{ }^{13} \mathrm{C}-\mathrm{NMR}, \mathrm{DEPT}$ : $\left(76 \mathrm{MHz}, \mathrm{CDCl}_{3}\right) \delta / \mathrm{ppm}=199.3(\mathrm{C}=\mathrm{O}), 135.8\left(\mathrm{CH}_{\mathrm{Ar}}\right)$, $134.1\left(\mathrm{C}_{\mathrm{Ar}}\right), 134.1\left(\mathrm{C}_{\mathrm{Ar}}\right), 129.7\left(\mathrm{CH}_{\mathrm{Ar}}\right), 127.7\left(\mathrm{CH}_{\mathrm{Ar}}\right), 68.9$ $\left(\mathrm{CH}_{2}\right), 51.3\left(\mathrm{CH}_{2}\right), 40.9\left(\mathrm{CH}_{2}\right), 33.3(\mathrm{CH}), 28.8(\mathrm{CH}), 27.0$ $\left(\mathrm{CH}_{3}\right), 23.4\left(\mathrm{CH}_{2}\right), 20.4\left(\mathrm{CH}_{3}\right), 19.4\left(\mathrm{C}_{\mathrm{q}}\right), 17.6\left(\mathrm{CH}_{3}\right), 14.9$ $\left(\mathrm{CH}_{3}\right)$. EI-MS $(70 \mathrm{eV}): m / z=386(30), 385\left(\left[\mathrm{M}-{ }^{\mathrm{t}} \mathrm{Bu}\right]^{+}\right.$, 100), 323 (31), 243 (44), 199 (92), 183 (50), 181 (35), 135 (41), 83 (35), 55 (36).

\section{Preparation of (3R,5S)-6-((tert-Butyldiphenylsilyl) oxy)-3,5-dimethylhexan-1-ol (5)}

To a solution of 4 (0.90 mmol, $400 \mathrm{mg}, 1$ eq.) in $\mathrm{CH}_{2} \mathrm{Cl}_{2}$ $(10 \mathrm{~mL})$ was added diisobutylaluminium hydride ( $1.17 \mathrm{mmol}, 1.3$ eq., solution in cyclohexane) at $-50{ }^{\circ} \mathrm{C}$ under a nitrogen atmosphere. After stirring for $17 \mathrm{~h}$ the reaction mixture was allowed to warm up to room temperature. The reaction was quenched by addition of saturated Rochelle solution $(10 \mathrm{~mL})$ and stirred for $30 \mathrm{~min}$ at room temperature. The phases were separated and the aqueous phase was extracted three times with $\mathrm{CH}_{2} \mathrm{Cl}_{2}(30 \mathrm{~mL})$. The combined organic phases were dried over $\mathrm{Na}_{2} \mathrm{SO}_{4}$ and the solvent removed under reduced pressure to yield the crude aldehyde. The reduction procedure was repeated and the obtained residue was purified by column chromatography (pentane/diethyl ether; 1:1) to afford alcohol $\mathbf{1 3}$ as a slightly yellow oil ( $267 \mathrm{mg}, 77 \%)$. In contrast to the published procedure (Horst et al. 2007) a two-step reduction proved to be necessary to obtain good yields. $[\alpha]_{\mathrm{D}}^{20}=-3.7$ $\left(10 \mathrm{mg} / \mathrm{mL} ; \mathrm{CHCl}_{3}\right)$. FT-IR: $\nu / \mathrm{cm}^{-1}=2928,2859,1466$, 1428, 1385, 1107, 1007, 821, 739, 700, 614. ${ }^{1} \mathrm{H}-\mathrm{NMR}$ : $\left(300 \mathrm{MHz}, \mathrm{CDCl}_{3}\right) \delta / \mathrm{ppm}=7.69(\mathrm{~m}, 4 \mathrm{H}), 7.48-7.36(\mathrm{~m}$, $6 \mathrm{H}), 3.74-3.58(\mathrm{~m}, 2 \mathrm{H}), 3.57-3.40(\mathrm{~m}, 2 \mathrm{H}), 1.86-1.68(\mathrm{~d}$, $J=6.6 \mathrm{~Hz}, 1 \mathrm{H}), 1.68-1.52(\mathrm{~m}, 2 \mathrm{H}), 1.49-1.22(\mathrm{~m}, 3 \mathrm{H}) .{ }^{13} \mathrm{C}-$ NMR, DEPT: $\left(76 \mathrm{MHz}, \mathrm{CDCl}_{3}\right) \delta / \mathrm{ppm}=135.8(\mathrm{CH}), 135.8$ (C), $134.2(\mathrm{CH}), 129.6(\mathrm{CH}), 127.7(\mathrm{CH}), 68.9\left(\mathrm{CH}_{2}\right), 61.2$ $\left(\mathrm{CH}_{2}\right), 41.3\left(\mathrm{CH}_{2}\right), 39.9\left(\mathrm{CH}_{2}\right), 33.2\left(\mathrm{CH}_{3}\right), 27.1\left(\mathrm{CH}_{3}\right), 27.0$ $\left(\mathrm{CH}_{3}\right), 20.4\left(\mathrm{CH}_{3}\right), 19.4\left(\mathrm{C}_{\mathrm{q}}\right), 17.8\left(\mathrm{CH}_{3}\right)$. EI-MS $(70 \mathrm{eV})$ : $m / z=327\left(\left[M-{ }^{t} \mathrm{Bu}\right]^{+}, 2\right), 200(11), 199$ (63), 181 (14), 139 (11), 135 (10), 111 (37), 69 (100), 57 (10), 55 (39), 41 (19).

\section{Preparation of (3R,5S)-6-((tert-Butyldiphenylsilyl) oxy)-3,5-dimethylhexyl 4-Methylbenzenesulfonate (6)}

Tosyl chloride ( $1.35 \mathrm{mmol}, 257 \mathrm{mg}, 2$ eq.) was added to a solution of 5 ( $0.67 \mathrm{mmol}, 259 \mathrm{mg}, 1$ eq. $)$ and pyridine ( $1.35 \mathrm{mmol}, 109 \mu \mathrm{L}, 2$ eq.) in $\mathrm{CH}_{2} \mathrm{Cl}_{2}(5 \mathrm{~mL})$. The reaction mixture was stirred at room temperature for $19 \mathrm{~h}$ under 
a nitrogen atmosphere. The solvent was removed under reduced pressure and the resulting residue was purified by column chromatography (pentane/diethyl ether; 20:1) to afford 6 as a colorless oil $(289 \mathrm{mg}, 80 \%)$. $[\alpha]_{\mathrm{D}}^{20}=-4.2$ $\left(10 \mathrm{mg} / \mathrm{mL} ; \mathrm{CHCl}_{3}\right)$. FT-IR: $\nu / \mathrm{cm}^{-1}=2955,2923,2854$, 1463, 1428, 1389, 1377, 1362, 1110, 1007, 999, 824, 795, 738, 700, 671, 665, 614, 528. ${ }^{1} \mathrm{H}-\mathrm{NMR}:\left(300 \mathrm{MHz}, \mathrm{CDCl}_{3}\right)$ $\delta / \mathrm{ppm}=7.81-7.75(\mathrm{~m}, 2 \mathrm{H}), 7.70-7.63(\mathrm{~m}, 4 \mathrm{H}), 7.48-7.27$ (m, 8H), 4.12-3.96 (m, 2H), $3.42(\mathrm{ddd}, J=16.1,9.8,5.9 \mathrm{~Hz}$, $2 \mathrm{H}), 2.43(\mathrm{~s}, 3 \mathrm{H}), 1.77-1.44(\mathrm{~m}, 3 \mathrm{H}), 1.32$ (ddt, $J=13.5$, $10.8,6.6 \mathrm{~Hz}, 3 \mathrm{H}), 1.05$ (s, 9H), 0.99-0.81 (m, 4H), 0.77 $(\mathrm{d}, J=6.5 \mathrm{~Hz}, 3 \mathrm{H}) \cdot{ }^{13} \mathrm{C}-\mathrm{NMR}$, DEPT: $\left(76 \mathrm{MHz}, \mathrm{CDCl}_{3}\right) \delta /$ $\mathrm{ppm}=144.7\left(\mathrm{C}_{\mathrm{Ar}}\right), 135.7\left(\mathrm{CH}_{\mathrm{Ar}}\right), 134.1\left(\mathrm{C}_{\mathrm{Ar}}\right), 133.4\left(\mathrm{C}_{\mathrm{Ar}}\right)$, $129.9\left(\mathrm{CH}_{\mathrm{Ar}}\right), 129.7\left(\mathrm{CH}_{\mathrm{Ar}}\right), 128.0\left(\mathrm{CH}_{\mathrm{Ar}}\right), 127.7\left(\mathrm{CH}_{\mathrm{Ar}}\right)$, 69.2 $\left(\mathrm{CH}_{2}\right), 68.8\left(\mathrm{CH}_{2}\right), 41.1\left(\mathrm{CH}_{2}\right), 35.7\left(\mathrm{CH}_{2}\right), 33.1(\mathrm{CH})$, $27.0\left(\mathrm{CH}_{3}\right), 27.0(\mathrm{CH}), 21.7\left(\mathrm{CH}_{3}\right), 19.9\left(\mathrm{C}_{\mathrm{q}}\right), 19.4\left(\mathrm{CH}_{3}\right)$, $17.7\left(\mathrm{CH}_{3}\right)$. EI-MS $(70 \mathrm{eV}): \mathrm{m} / z=353(35), 293(66), 199$ (52), 181 (20), 135 (21), 111 (58), 69 (100), 91 (51), (48), 41 (28).

\section{Preparation of (2S,4S)-tert-Butyl(2,4-dimethylheptadecyl) oxydiphenylsilane (7)}

1-Bromoundecane was added $(2.13 \mathrm{mmol}, 500 \mathrm{mg})$ to a mixture of magnesium turnings $(2.55 \mathrm{mmol}, 62 \mathrm{mg})$ in THF $(10 \mathrm{~mL})$. The reaction mixture was heated to reflux for $30 \mathrm{~min}$, then cooled to room temperature. Compound 6 (0.51 mmol, $273 \mathrm{mg}, 1$ eq.) and $\mathrm{CuBr} \cdot \mathrm{SMe}_{2}(0.10 \mathrm{mmol}$, $21 \mathrm{mg}, 0.2$ eq. $)$ were dissolved in THF $(6 \mathrm{~mL})$ under a nitrogen atmosphere. The freshly prepared Grignard solution was added at $0{ }^{\circ} \mathrm{C}$. After warming to room temperature, the reaction mixture was stirred for $1 \mathrm{~h}$. After quenching with saturated $\mathrm{NH}_{4} \mathrm{Cl}$ solution $(6 \mathrm{~mL})$ the phases were separated and the aqueous phase was extracted three times with diethyl ether $(30 \mathrm{~mL})$. The combined organic phases were dried over $\mathrm{Na}_{2} \mathrm{SO}_{4}$ and the solvent was removed under reduced pressure. The residue was purified by column chromatography (pentane/diethyl ether; 20:1) to afford 7 as a colorless oil $(113 \mathrm{mg}, 42 \%)$. $[\alpha]_{\mathrm{D}}^{20}=-3.3\left(10 \mathrm{mg} / \mathrm{mL} ; \mathrm{CHCl}_{3}\right)$. FT-IR: $\nu / \mathrm{cm}^{-1}=3067,2957,2929,2860,2323,1596$, $1465,1429,1361,1300,1258,1213,1180,1103,945,892$, 816, 744, 701, 663, 614, 578, 555. ${ }^{1} \mathrm{H}-\mathrm{NMR}:(300 \mathrm{MHz}$, $\left.\mathrm{CDCl}_{3}\right) \delta / \mathrm{ppm}=7.73-7.63(\mathrm{~m}, 4 \mathrm{H}), 7.50-7.31(\mathrm{~m}, 6 \mathrm{H})$, 3.47 (ddd, $J=16.2,9.8,5.9 \mathrm{~Hz}, 2 \mathrm{H}), 1.74$ (d, $J=6.7 \mathrm{~Hz}$, 1H), 1.50-1.19 (m, 25H), 1.10-1.03 (m, 9H), 0.96-0.85 (m, $7 \mathrm{H}), 0.82(\mathrm{~d}, J=6.4 \mathrm{~Hz}, 3 \mathrm{H}) .{ }^{13} \mathrm{C}-\mathrm{NMR}$, DEPT: $(76 \mathrm{MHz}$, $\left.\mathrm{CDCl}_{3}\right) \delta / \mathrm{ppm}=135.8\left(\mathrm{CH}_{\mathrm{Ar}}\right), 134.3\left(\mathrm{C}_{\mathrm{Ar}}\right), 129.6\left(\mathrm{CH}_{\mathrm{Ar}}\right)$, $127.7\left(\mathrm{CH}_{\mathrm{Ar}}\right), 69.1\left(\mathrm{CH}_{2}\right), 41.3\left(\mathrm{CH}_{2}\right), 37.0\left(\mathrm{CH}_{2}\right), 33.3$ $(\mathrm{CH}), 32.1\left(\mathrm{CH}_{2}\right), 30.2\left(\mathrm{CH}_{2}\right), 30.2(\mathrm{CH}), 29.9\left(\mathrm{CH}_{2}\right), 29.9$ $\left(\mathrm{CH}_{2}\right), 29.8\left(\mathrm{CH}_{2}\right), 29.5\left(\mathrm{CH}_{2}\right), 27.0\left(\mathrm{CH}_{3}\right), 27.0\left(\mathrm{CH}_{2}\right)$, $22.9\left(\mathrm{CH}_{2}\right), 20.5\left(\mathrm{CH}_{3}\right), 19.5\left(\mathrm{C}_{\mathrm{q}}\right), 17.9\left(\mathrm{CH}_{3}\right), 14.3\left(\mathrm{CH}_{3}\right)$.
EI-MS (70 eV): $m / z=465$ (34), 200 (19), 199 (100), 97 (23), 83 (25), 83 (19), 69 (28), 57 (40), 55 (20), 43 (37).

\section{Preparation of (2S,4S)-2,4-Dimethylheptadecan-1-ol (8)}

TBAF ( $0.57 \mathrm{mmol}, 574 \mu \mathrm{L}, 3$ eq.) was added to a solution of 7 ( $0.19 \mathrm{mmol}, 100 \mathrm{mg}, 1$ eq.) in THF ( $5 \mathrm{~mL})$. The reaction mixture was stirred at room temperature for $24 \mathrm{~h}$ under a nitrogen atmosphere. The solvent was removed under reduced pressure and the residue was purified by column chromatography (pentane/diethyl ether; 20:1) to afford 8 as a colorless oil with traces of siloxanes as impurities $\left(21 \mathrm{mg}\right.$, crude). $[\alpha]_{\mathrm{D}}^{20}=-37.6\left(10 \mathrm{mg} / \mathrm{mL} ; \mathrm{CHCl}_{3}\right)$. FT-IR: $\nu / \mathrm{cm}^{-1}=3341,2955,2922,2853,1462,1377,1112,1036$, 987, 865, 821, 704, 606. ${ }^{1} \mathrm{H}-\mathrm{NMR}:\left(600 \mathrm{MHz}, \mathrm{CDCl}_{3}\right) \delta$ $3.49-3.27(\mathrm{~m}, 2 \mathrm{H}), 1.70-1.60(\mathrm{~m}, 1 \mathrm{H}), 1.46-1.37(\mathrm{~m}, 1 \mathrm{H})$, $1.27-1.10(\mathrm{~m}, 26 \mathrm{H}), 1.09-0.91(\mathrm{~m}, 2 \mathrm{H}), 0.90-0.75(\mathrm{~m}$, 10H). ${ }^{13} \mathrm{C}-\mathrm{NMR}$, DEPT: $(151 \mathrm{MHz}, \mathrm{CDCl} 3) \delta / \mathrm{ppm}=68.2$ $\left(\mathrm{CH}_{2}\right), 40.9\left(\mathrm{CH}_{2}\right), 36.5\left(\mathrm{CH}_{2}\right), 32.9(\mathrm{CH}), 31.7\left(\mathrm{CH}_{2}\right), 29.9$ (CH), $29.8\left(\mathrm{CH}_{2}\right), 29.5\left(\mathrm{CH}_{2}\right), 29.5\left(\mathrm{CH}_{2}\right), 29.5\left(\mathrm{CH}_{2}\right), 29.2$ $\left(\mathrm{CH}_{2}\right), 26.7\left(\mathrm{CH}_{2}\right), 22.5\left(\mathrm{CH}_{2}\right), 20.2\left(\mathrm{CH}_{3}\right), 17.1\left(\mathrm{CH}_{3}\right), 13.9$ $\left(\mathrm{CH}_{3}\right)$. EI-MS $(70 \mathrm{eV}): m / z=283(<1), 266(<1), 224(10)$, 209 (6), 196 (7), 168 (7), 111 (22), 97 (31), 83 (100), 71 (44), 70 (39), 69 (52), 57 (97), 56 (60), 55 (85), 43 (81), $41(61)$.

\section{Preparation of (2S,4S)-2,4-Dimethylheptadecanoic Acid (9)}

Under a nitrogen atmosphere, $\mathrm{RuCl}_{3}(0.014 \mathrm{mmol}, 3 \mathrm{mg}$, 0.3 eq.) and $\mathrm{NaIO}_{4}(0.281 \mathrm{mmol}, 60 \mathrm{mg}, 5$ eq. $)$ were added to a mixture of crude $8(0.056 \mathrm{mmol}, 16 \mathrm{mg}, 1$ eq. $), \mathrm{H}_{2} \mathrm{O}$ $(1.2 \mathrm{~mL}), \mathrm{CH}_{3} \mathrm{CN}(1.2 \mathrm{~mL})$ and $\mathrm{CCl}_{4}(2.4 \mathrm{~mL})$. The mixture was stirred at room temperature for $3.5 \mathrm{~h}$. After addition of $\mathrm{CH}_{2} \mathrm{Cl}_{2}(4 \mathrm{~mL})$ and $\mathrm{H}_{2} \mathrm{O}(1 \mathrm{~mL})$ the phases were separated and the aqueous phase was extracted three times with $\mathrm{CH}_{2} \mathrm{Cl}_{2}(10 \mathrm{~mL})$. The combined organic phases were dried over $\mathrm{Na}_{2} \mathrm{SO}_{4}$. The solvent was removed under reduced pressure and the residue was purified by column chromatography (pentane/ethyl acetate/acetic acid; 90:10:1) to afford acid 9 as a colorless oil $(11 \mathrm{mg}, 66 \%)$. $[\alpha]_{\mathrm{D}}^{20}=+7.0$ $\left(10 \mathrm{mg} / \mathrm{mL} ; \mathrm{CHCl}_{3}\right)$. FT-IR: $\nu / \mathrm{cm}^{-1}=2956,2923,2853$, 1815, 1707, 1464, 1416, 1379, 1290, 1235, 1091, 1018, 947, 810, 722, 529. ${ }^{1} \mathrm{H}-\mathrm{NMR}:\left(600 \mathrm{MHz}, \mathrm{CDCl}_{3}\right) \delta / \mathrm{ppm}=9.58$ $(\mathrm{d}, J=2.5 \mathrm{~Hz}, 1 \mathrm{H}), 2.67-2.51(\mathrm{~m}, 1 \mathrm{H}), 1.80-1.66(\mathrm{~m}, 1 \mathrm{H})$, $1.53-1.41(\mathrm{~m}, 1 \mathrm{H}), 1.40-1.02(\mathrm{~m}, 31 \mathrm{H}), 0.95-0.79(\mathrm{~m}, 7 \mathrm{H})$. ${ }^{13} \mathrm{C}-\mathrm{NMR}, \mathrm{DEPT}:\left(151 \mathrm{MHz}, \mathrm{CDCl}_{3}\right) \delta / \mathrm{ppm}=172.7(\mathrm{C})$, $41.4\left(\mathrm{CH}_{2}\right), 37.2\left(\mathrm{CH}_{2}\right), 32.1\left(\mathrm{CH}_{2}\right), 30.9\left(\mathrm{CH}_{2}\right), 30.1(\mathrm{CH})$, $29.9\left(\mathrm{CH}_{2}\right), 29.5\left(\mathrm{CH}_{2}\right), 26.9\left(\mathrm{CH}_{2}\right), 22.9\left(\mathrm{CH}_{2}\right), 19.7\left(\mathrm{CH}_{3}\right)$, $18.0\left(\mathrm{CH}_{3}\right), 14.27\left(\mathrm{CH}_{3}\right)$. A small portion was converted into the respective methyl ester by treatment with trimethylsilyldiazomethane. EI-MS $(70 \mathrm{eV}): m / z=312\left([M]^{+}, 3\right), 241$ 
(12), 129 (7), 101 (59), 88 (100), 71 (7), 69 (12), 57 (13), 55 (12), 43 (12), 41 (10).

\section{Preparation of Tetradecyl (2S,4S)-2,4-Dimethylheptadecanoate (10)}

1-Tetradecanol ( $0.007 \mathrm{mmol}, 0.53 \mathrm{mg}, 1$ eq., $1 \% \mathrm{wt}$ in DCM), dicyclohexylcarbodiimide $(0.007 \mathrm{mmol}, 1.28 \mathrm{mg}, 1$ eq., $1 \% \mathrm{wt}$ in DCM) and 4-dimethylaminopyridine $(0.007 \mathrm{mmol}$, $1.38 \mathrm{mg}, 1$ eq., $1 \% \mathrm{wt}$ in DCM) were added to a solution of $(2 S, 4 S)$-2,4-dimethylheptadecanoic acid (9) $(0.007 \mathrm{mmol}$, $2 \mathrm{mg}, 1$ eq.) in DCM (5 mL). After stirring for $20 \mathrm{~h}$ at room temperature the solvent was removed under reduced pressure and the residue was purified by column chromatography (pentane/diethyl ether; 100:1) to afford $\mathbf{1 1}$ as a colorless oil $(2 \mathrm{mg}, 58 \%) \cdot[\alpha]_{\mathrm{D}}^{20}=+8.0\left(2 \mathrm{mg} / \mathrm{mL} ; \mathrm{CHCl}_{3}\right)$. FT-IR: $\nu /$ $\mathrm{cm}^{-1}=2923,2855,2319,1736,1461,1372,1172,673,603$, 565, 546. ${ }^{1} \mathrm{H}-\mathrm{NMR}:\left(500 \mathrm{MHz}, \mathrm{CDCl}_{3}\right) \delta / \mathrm{ppm}=4.11-3.99$ $(\mathrm{m}, 2 \mathrm{H}), 2.58-2.46(\mathrm{~m}, 1 \mathrm{H}), 1.74-1.66(\mathrm{~m}, 1 \mathrm{H}), 1.65-1.57$ $(\mathrm{m}, 3 \mathrm{H}), 1.42-1.17(\mathrm{~m}, 38 \mathrm{H}), 1.16-1.05(\mathrm{~m}, 5 \mathrm{H}), 0.91-0.78$ $(\mathrm{m}, 10 \mathrm{H}) .{ }^{13} \mathrm{C}-\mathrm{NMR}$, DEPT: $\left(126 \mathrm{MHz}, \mathrm{CDCl}_{3}\right) \delta /$ ppm $=177.4\left(\mathrm{C}_{\mathrm{q}}\right), 64.4\left(\mathrm{CH}_{2}\right), 41.8\left(\mathrm{CH}_{2}\right), 37.7(\mathrm{CH}), 37.3$ $\left(\mathrm{CH}_{2}\right), 32.1\left(\mathrm{CH}_{2}\right), 31.0(\mathrm{CH}), 30.1\left(\mathrm{CH}_{2}\right), 29.9\left(\mathrm{CH}_{2}\right), 29.8$ $\left(\mathrm{CH}_{2}\right), 29.8\left(\mathrm{CH}_{2}\right), 29.7\left(\mathrm{CH}_{2}\right), 29.5\left(\mathrm{CH}_{2}\right), 29.4\left(\mathrm{CH}_{2}\right), 28.9$ $\left(\mathrm{CH}_{2}\right), 27.0\left(\mathrm{CH}_{2}\right), 26.1\left(\mathrm{CH}_{2}\right), 22.9\left(\mathrm{CH}_{2}\right), 19.7\left(\mathrm{CH}_{3}\right), 18.2$ $\left(\mathrm{CH}_{3}\right), 14.3\left(\mathrm{CH}_{3}\right)$. EI-MS $(70 \mathrm{eV}): \mathrm{m} / \mathrm{z}(\%)=299(100), 241$ (17), 196 (23), 111 (14), 97 (24), 87 (50), 83 (25), 75 (30), 74 (61), 71 (36), 69 (31), 57 (55), 55 (57), 43 (41), 41 (17).

\section{Results}

\section{Analysis of Spider Extracts by GC/MS}

Dichloromethane extracts of silk from females and of the cuticle of both sexes of Argiope bruennichi were individually analyzed by GC/MS and the average relative proportions of the compounds were determined (Table 1). The samples were then sex-specifically combined to allow identification of minor components by GC/MS (Fig. 2). The female-produced sex pheromone, trimethyl methylcitrate (I 1523), as well as 3-octanoyloxy- $\gamma$-butyrolactone, a compound of unknown function specific to silk from females (Chinta et al. 2010), were the only compounds eluting early. Besides some hydrocarbons, the dominant cuticular lipids showed mass spectra consistent with waxtype esters (McLafferty and Turecek 1993; Chinta et al. 2016). These spectra are dominated by ions formed in a characteristic McLafferty rearrangement that cleaves the O-alkyl bond and, together with hydrogen transfer, leads to the protonated acid and alkene ions.

\section{Statistical Analyses}

We then performed discriminant analyses (DAs). To reduce overfitting due to a large influence of minor components, only peaks that were present in more than $1 \%$ in all samples were used. These included three hydrocarbons (I $2700,2900,3061)$ and four wax esters $(3273,3380,3476$, 3575). For all seven compounds (Wilk's lambda $=0.037$, Chi2 $=215.9, \mathrm{p}<0.0001$, d.f. $=12$ ), the three hydrocarbons (Wilk's lambda $=0.27$, Chi2 $=88.9, \mathrm{p}<0.0001$, d.f. $=4$ ), and the four wax esters (Wilk's lambda $=0.088$, Chi $2=163.0, p<0.0001$, d.f. $=6$ ) the DAs significantly separated the three sample types (Fig. 3). In all analyses, most of the observed variation is explained by the first discriminant function, separating male from female samples, while cuticular and web silk samples from females were mostly separated by the second function and generally grouped closer together.

\section{Identification of Wax Esters}

The wax esters were identified by interpretation of their mass spectra, derivatization procedures, and synthesis. Because many different esters were present, we will illustrate the identification procedure by one example.

The mass spectrum of the peak with a retention index $I$ of 3273 in samples from both males and females (Fig. 4) showed a molecular ion of $\mathrm{m} / \mathrm{z} 494$ and a large peak at $\mathrm{m} / \mathrm{z}$ 313 , likely the protonated acid ion $\mathrm{C}_{20} \mathrm{H}_{41} \mathrm{O}_{2}{ }^{+}$. The ion $\mathrm{m} / z, 182$ (likely $\mathrm{C}_{13} \mathrm{H}_{26}{ }^{+}$) corresponds to the alcohol part. Additionally, smaller peaks at $\mathrm{m} / \mathrm{z} 299$ and 327 were present, along with $\mathrm{m} / z 196$ and 168. These peak pairs indicate that three esters elute together, consisting of $\mathrm{C}_{19}$-acid and $\mathrm{C}_{14^{-}}$-alcohol, $\mathrm{C}_{20^{-}}$acid and $\mathrm{C}_{13^{-}}$-alcohol, and $\mathrm{C}_{21}$-acid and $\mathrm{C}_{12}$-alcohol. A characteristic ion at $\mathrm{m} / \mathrm{z} 74$ proved a methyl group at C-2 of the acid (Chinta et al. 2016).

To identify the number and position of methyl groups in the chains, microreactions with the extracts were performed. The natural samples were transesterified with TMSH to the corresponding methyl esters and free alcohols (Müller et al. 1990). The methyl esters allow determination of methyl group positions near the carbonyl group (Ryhage and Stenhagen 1960a). These esters were then again transesterified with sodium 3-pyridinylmethoxide to form the corresponding 3-pyridinylmethyl esters, while the free alcohols were esterified under Steglich conditions with nicotinic acid to the corresponding nicotinates. All these derivatized extracts were analyzed by GC/MS and the data were used to identify the number and position of methyl branches in both acids and alcohols (Fig. 1 and Tables S1 and S2 in the SI).

Methyl esters display characteristic fragment ions formed by $\beta$-cleavage and McLafferty rearrangement. Because $\mathrm{C}-2$ substituents are included into the rearrangement, 
Table 1 LIST OF COMPOUNDS DETECTED

IN EXTRACTS OF $A$. $B R U E N N I C H I$. Individual samples of 30 bodies and webs of females, as well as samples from 20 bodies of males, were analyzed. Compounds often coeluted within one peak. The average percentage of each peak within the whole sample is reported. Peaks are separated with lines in the table. Major components of peak groups are marked in bold. The three most abundant compounds of each sample type are also marked in bold in the columns on the right

\begin{tabular}{|c|c|c|c|c|c|c|c|}
\hline \multirow[b]{2}{*}{$I$} & \multirow[b]{2}{*}{ Substance } & \multicolumn{2}{|c|}{ Female body } & \multicolumn{2}{|c|}{ Female web } & \multicolumn{2}{|c|}{ Male body } \\
\hline & & mean $\%$ & $\mathrm{SD}$ & mean $\%$ & $\mathrm{SD}$ & mean $\%$ & $\mathrm{SD}$ \\
\hline 1523 & Trimethyl methylcitrate & 0.84 & 0.77 & 0.95 & 1.17 & - & \\
\hline 1803 & 3-Octanoyloxy- $\gamma$-butyrolactone & - & & 3.07 & 2.83 & - & \\
\hline 1900 & Nonadecane & $\operatorname{tr}$ & & 0.20 & 0.18 & - & \\
\hline 1946 & Hexadecenoic acid & 0.08 & 0.30 & - & & 一 & \\
\hline 1959 & Hexadecanoic acid & 0.08 & 0.14 & 1.33 & 1.65 & 0.17 & 0.12 \\
\hline 1992 & $\begin{array}{l}\text { Ethyl hexadecanoate } \\
\text { Eicosane }\end{array}$ & $\operatorname{tr}$ & & 0.65 & 0.29 & - & \\
\hline 2090 & Nonadecanal & 0.10 & 0.13 & $\operatorname{tr}$ & & - & \\
\hline 2100 & Heneicosane & 0.10 & 0.07 & 1.25 & 0.44 & & \\
\hline 2142 & $\begin{array}{l}\text { Octadecadienoic acid } \\
\text { Octadecenoic acid }\end{array}$ & $\operatorname{tr}$ & & - & & $\operatorname{tr}$ & \\
\hline 2160 & $\begin{array}{l}\text { Octadecanoic acid } \\
\text { Ethyl octadecenoate }\end{array}$ & $\operatorname{tr}$ & & 0.68 & 1.02 & 0.22 & 0.54 \\
\hline 2193 & $\begin{array}{l}\text { Ethyl octadecanoate } \\
\text { Docosane }\end{array}$ & 0.09 & 0.06 & 1.50 & 0.57 & - & \\
\hline 2209 & Unknown & $\operatorname{tr}$ & & - & & - & \\
\hline 2259 & 2-Methyldocosane & 0.07 & 0.04 & 0.91 & 0.47 & - & \\
\hline 2276 & Tricosene & 0.07 & 0.14 & 0.07 & 0.12 & - & \\
\hline 2283 & Tricosene & $\operatorname{tr}$ & & - & & - & \\
\hline 2300 & $\begin{array}{l}\text { Tricosane } \\
\text { 1-Eicosanol }\end{array}$ & 1.56 & 1.01 & 3.00 & 1.03 & 0.24 & 0.18 \\
\hline 2334 & 9-Methyltricosane & 0.07 & 0.05 & 0.49 & 0.30 & - & \\
\hline 2339 & 7-Methyltricosane & & & & & & \\
\hline 2348 & 5-Methyltricosane & $\operatorname{tr}$ & & $\operatorname{tr}$ & & - & \\
\hline 2371 & 3-Methyltricosane & $\operatorname{tr}$ & & 0.23 & 0.18 & - & \\
\hline 2400 & Tetracosane & 0.13 & 0.08 & 1.59 & 0.57 & $\operatorname{tr}$ & \\
\hline 2459 & 2-Methyltetracosane & 0.15 & 0.07 & 0.36 & 0.25 & 0.06 & 0.11 \\
\hline 2470 & 3-Methyltetracosane & $\operatorname{tr}$ & & 0.18 & 0.18 & - & \\
\hline 2474 & Pentacosene & $\operatorname{tr}$ & & - & & - & \\
\hline 2483 & Pentacosene & $\operatorname{tr}$ & & - & & - & \\
\hline 2500 & Pentacosane & 3.74 & 1.71 & 3.30 & 1.19 & 0.99 & 0.37 \\
\hline 2527 & 11-Methylpentacosane + impurity $^{1}$ & & & - & & - & \\
\hline 2531 & 9-Methylpentacosane + impurity ${ }^{1}$ & & & & & & \\
\hline 2538 & 7-Methylpentacosane & $\operatorname{tr}$ & & - & & $\operatorname{tr}$ & \\
\hline 2547 & 5-Methylpentacosane & $\operatorname{tr}$ & & 0.09 & 0.18 & - & \\
\hline 2558 & 2-Methylpentacosane & $\operatorname{tr}$ & & 0.17 & 0.18 & $\operatorname{tr}$ & \\
\hline 2571 & 3-Methylpentacosane & $\operatorname{tr}$ & & 0.28 & 0.23 & - & \\
\hline 2592 & Unknown & $\operatorname{tr}$ & & - & & & \\
\hline 2600 & Hexacosane & 0.16 & 0.06 & 1.08 & 0.42 & $\operatorname{tr}$ & \\
\hline 2628 & Unknown & 0.09 & 0.07 & 0.58 & 0.51 & 2.80 & 1.04 \\
\hline 2658 & 2-Methylhexacosane & 0.19 & 0.11 & 0.64 & 0.48 & - & \\
\hline 2677 & Heptacosene & $\operatorname{tr}$ & & - & & - & \\
\hline 2700 & Heptacosane + impurity & 3.76 & 1.32 & 5.99 & 2.04 & 1.13 & 0.45 \\
\hline 2733 & 7-Methylheptacosane + impurity $^{1}$ & & & - & & - & \\
\hline 2748 & 5-Methylheptacosane & $\operatorname{tr}$ & & - & & - & \\
\hline 2758 & Hydrocarbon & $\operatorname{tr}$ & & 0.47 & 0.59 & - & \\
\hline 2771 & 3-Methylheptacosane & 0.12 & 0.08 & 0.24 & 0.35 & 0.28 & 0.32 \\
\hline 2830 & Unknown & 0.21 & 0.17 & - & & 4.13 & 1.96 \\
\hline 2842 & Unknown & $\operatorname{tr}$ & & - & & - & \\
\hline
\end{tabular}


Table 1 (continued)

\begin{tabular}{|c|c|c|c|c|c|c|c|}
\hline \multirow[b]{2}{*}{$I$} & \multirow[b]{2}{*}{ Substance } & \multicolumn{2}{|c|}{ Female body } & \multicolumn{2}{|c|}{ Female web } & \multicolumn{2}{|c|}{ Male body } \\
\hline & & mean $\%$ & SD & mean $\%$ & SD & mean $\%$ & SD \\
\hline 2860 & 2-Methyloctacosane & 1.03 & 0.60 & 0.54 & 0.55 & 1.46 & 0.61 \\
\hline 2871 & 3-Methyloctacosane & $\operatorname{tr}$ & & 0.17 & 0.21 & - & \\
\hline 2900 & Nonacosane & 2.23 & 0.82 & 2.55 & 0.60 & 1.00 & 0.40 \\
\hline 2905 & Unknown & $\operatorname{tr}$ & & 0.30 & 0.98 & - & \\
\hline 2929 & 15-Methylnonacosane & 1.31 & 0.80 & 2.08 & 1.36 & 0.41 & 0.38 \\
\hline 2933 & $\begin{array}{l}\text { 13-Methylnonacosane } \\
\text { 11-Methylnonacosane } \\
\text { 9-Methylnonacosane }\end{array}$ & & & & & & \\
\hline 2938 & 7-Methylnonacosane & 0.69 & 0.39 & 0.44 & 0.37 & $\operatorname{tr}$ & \\
\hline 2948 & 5-Methylnonacosane & 0.17 & 0.10 & 0.10 & 0.20 & - & \\
\hline 2955 & Hydrocarbon & $\operatorname{tr}$ & & - & & - & \\
\hline 2960 & Hydrocarbon & 0.09 & 0.09 & $\operatorname{tr}$ & & 0.12 & 0.25 \\
\hline 2966 & 7,11-Dimethylnonacosane & $\operatorname{tr}$ & & - & & $\operatorname{tr}$ & \\
\hline 2971 & 3-Methylnonacosane & 0.11 & 0.06 & 2.14 & 1.62 & 0.23 & 0.26 \\
\hline 2978 & 5,9-Dimethylnonacosane & $\operatorname{tr}$ & & - & & 0.15 & 0.19 \\
\hline 3000 & Triacontane & 0.20 & 0.10 & 1.83 & 0.73 & - & \\
\hline 3008 & Hydrocarbon & $\operatorname{tr}$ & & 0.39 & 0.38 & - & \\
\hline 3030 & $\begin{array}{l}\text { 15-Methyltriacontane } \\
\text { 16-Methyltriacontane }\end{array}$ & 0.51 & 0.35 & 1.83 & 2.72 & 0.70 & 0.39 \\
\hline 3042 & 6-Methyltriacontane & 0.08 & 0.07 & 0.23 & 0.28 & - & \\
\hline 3061 & 2-Methyltriacontane & 1.82 & 0.90 & 1.11 & 0.65 & 1.72 & 0.70 \\
\hline 3072 & Hydrocarbon & 0.10 & 0.12 & 0.32 & 0.36 & - & \\
\hline 3076 & $\begin{array}{l}\text { Tridecyl stearate } \\
\text { Dodecyl nonadecanoate }\end{array}$ & - & & - & & 1.59 & 0.54 \\
\hline 3091 & $\begin{array}{l}\text { 2,12-Dimethyltriacontane } \\
\text { 2,14-Dimethyltriacontane }\end{array}$ & 0.42 & 0.38 & 0.14 & 0.30 & - & \\
\hline 3101 & $\begin{array}{l}\text { 2,6-Dimethyltriacontane } \\
\text { 2,8-Dimethyltriacontane }\end{array}$ & 1.70 & 0.88 & 2.69 & 0.80 & 0.48 & 0.37 \\
\hline 3114 & Hydrocarbon & 0.25 & 0.29 & 0.35 & 0.42 & - & \\
\hline 3118 & Hydrocarbon & & & & & & \\
\hline 3122 & Hydrocarbon & 0.16 & 0.20 & 0.09 & 0.36 & - & \\
\hline 3129 & 15-Methylhentriacontane & 2.58 & 1.55 & 2.69 & 1.71 & - & \\
\hline 3135 & $\begin{array}{l}\text { 13-Methylhentriacontane } \\
\text { 11-Methylhentriacontane } \\
\text { 9-Methylhentriacontane }\end{array}$ & & & & & & \\
\hline 3139 & 7-Methylhentriacontane & 0.25 & 0.13 & 0.27 & 0.43 & - & \\
\hline 3160 & Hydrocarbon & 0.53 & 0.40 & 0.48 & 0.44 & - & \\
\hline 3166 & Hydrocarbon & 0.26 & 0.19 & - & & - & \\
\hline 3173 & $\begin{array}{l}\text { Tridecyl syn-2,4-dimethylheptadecanoate } \\
\text { 5,15-Dimethylhentriacontane }\end{array}$ & 0.29 & 0.24 & 0.22 & 0.41 & 14.88 & 2.44 \\
\hline 3176 & Tridecyl anti-2,4-dimethylheptadecanoate & & & & & & \\
\hline 3190 & Unknown & 0.06 & 0.09 & - & & - & \\
\hline 3199 & Tridecyl 2,4,8-trimethylheptadecanoate & 0.45 & 0.36 & 1.72 & 0.88 & 0.70 & 0.39 \\
\hline 3217 & $\begin{array}{l}\text { Tridecyl 2,4,14-trimethylheptadecanoate } \\
\text { Tetradecyl 2,4,14-trimethylhexadecanoate }\end{array}$ & - & & - & & 0.21 & 0.25 \\
\hline 3226 & 16-Methyldotriacontane & 0.43 & 0.28 & 1.91 & 2.00 & 0.89 & 0.22 \\
\hline 3230 & $\begin{array}{l}\text { 14-Methyldotriacontane } \\
\text { Tridecyl 2,4,16-trimethylheptadecanoate } \\
\text { Tetradecyl 2,4,16-trimethylhexadecanoate }\end{array}$ & & & & & & \\
\hline
\end{tabular}


Table 1 (continued)

\begin{tabular}{|c|c|c|c|c|c|c|c|}
\hline \multirow[b]{2}{*}{$I$} & \multirow[b]{2}{*}{ Substance } & \multicolumn{2}{|c|}{ Female body } & \multicolumn{2}{|c|}{ Female web } & \multicolumn{2}{|c|}{ Male body } \\
\hline & & mean $\%$ & $\mathrm{SD}$ & mean $\%$ & $\mathrm{SD}$ & mean $\%$ & $\mathrm{SD}$ \\
\hline 3256 & 6-Methyldotriacontane & 0.16 & 0.16 & - & & - & \\
\hline 3260 & 2-Methyldotriacontane & 0.20 & 0.12 & - & & - & \\
\hline 3273 & $\begin{array}{l}\text { Tetradecyl 2,4-dimethylheptadecanoate } \\
\text { Tridecyl 2,4-dimethyloctadecanoate } \\
\text { Dodecyl 2,4-dimethylnonadecanoate }\end{array}$ & 1.19 & 1.08 & 1.05 & 0.88 & 16.03 & 1.12 \\
\hline 3289 & Unknown & 0.86 & 0.58 & 0.34 & 0.44 & - & \\
\hline 3300 & Tritriacontane & 0.51 & 0.26 & 1.31 & 0.69 & - & \\
\hline 3316 & Unknown & 0.33 & 0.33 & 0.24 & 0.31 & - & \\
\hline 3323 & 17-Methyltritriacontane & 2.45 & 0.92 & 2.41 & 1.12 & - & \\
\hline 3329 & 11-Methyltritriacontane & & & & & & \\
\hline 3336 & $\begin{array}{l}\text { 9-Methyltritriacontane } \\
\text { Unknown }\end{array}$ & & & & & & \\
\hline 3330 & Tetradecyl 2,4,14-trimethylheptadecanoate & - & & - & & 0.35 & 0.33 \\
\hline 3352 & 7,11,15-Trimethyltritriacontane & 1.36 & 1.05 & 0.78 & 0.75 & 0.29 & 0.28 \\
\hline 3360 & 11,21-Dimethyltritriacontane & 0.35 & 0.33 & 0.13 & 0.29 & - & \\
\hline 3380 & $\begin{array}{l}\text { Tridecyl 2,4-dimethylnonadecanoate } \\
\text { Tetradecyl 2,4-dimethyloctadecanoate }\end{array}$ & 15.77 & 7.93 & 10.30 & 7.15 & 32.94 & 2.79 \\
\hline 3384 & Tridecyl 2,4,6-trimethylnonadecanoate & 0.72 & 0.72 & 0.17 & 0.39 & - & \\
\hline $\begin{array}{l}3397 \\
3405\end{array}$ & $\begin{array}{l}\text { Tridecyl 2,4,8-trimethylnonadecanoate } \\
\text { Tridecyl 2,4,10-trimethylnonadecanoate }\end{array}$ & 0.52 & 0.50 & 0.93 & 0.64 & 0.15 & 0.31 \\
\hline 3409 & Tridecyl 2,4,12-trimethylnonadecanoate & 0.09 & 0.15 & - & & - & \\
\hline 3416 & $\begin{array}{l}\text { Tetradecyl 2,4,14-trimethyloctadecanoate } \\
\text { Tridecyl 2,4,14-trimethylnonadecanoate }\end{array}$ & 0.33 & 0.13 & - & & 0.21 & 0.24 \\
\hline 3430 & $\begin{array}{l}\text { Tetradecyl 2,4,16-trimethylocatadecanoate } \\
\text { Tridecyl 2,4,16-trimethylnonadecanoate }\end{array}$ & 0.79 & 0.27 & 1.91 & 1.07 & 0.75 & 0.29 \\
\hline 3445 & $\begin{array}{l}\text { Tetradecyl trimethyloctadecanoate } \\
\text { Pentadecyl trimethylheptadecanoate }\end{array}$ & $\operatorname{tr}$ & & - & & - & \\
\hline 3476 & $\begin{array}{l}\text { Tetradecyl syn-2,4-dimethylnonadecanoate } \\
\text { Tridecyl syn-2,4-dimethylicosanoate } \\
\text { Dodecyl syn-2,4-dimethylhenicosanoate }\end{array}$ & 12.89 & 4.51 & 7.38 & 4.00 & 11.25 & 1.65 \\
\hline 3481 & $\begin{array}{l}\text { Tetradecyl anti-2,4-dimethylnonadecanoate } \\
\text { Tridecyl anti-2,4-dimethylicosanoate } \\
\text { Dodecyl anti-2,4-dimethylhenicosanoate }\end{array}$ & 0.67 & 0.64 & 0.28 & 0.81 & 0.22 & 0.29 \\
\hline 3495 & $\begin{array}{l}\text { Pentadecyl 2,4,6-trimethyloctadecanoate } \\
\text { Tetradecyl 2,4,6-trimethylnonadecanoate } \\
\text { Tridecyl 2,4,6-trimethylicosanoate }\end{array}$ & 0.14 & 0.18 & - & & - & \\
\hline 3500 & Wax ester & 0.13 & 0.13 & 0.71 & 0.51 & - & \\
\hline 3504 & Wax ester & $\operatorname{tr}$ & & - & & - & \\
\hline 3528 & $\begin{array}{l}\text { Tetradecyl 2,4,14-trimethylnonadecanoate } \\
\text { Pentadecyl 2,4,14-trimethyloctadecanoate } \\
\text { Hexadecyl 2,4,14-trimethylheptadecanoate }\end{array}$ & 0.96 & 0.29 & 1.93 & 0.96 & 0.06 & 0.20 \\
\hline 3544 & $\begin{array}{l}\text { Pentadecyl 2,4,16-trimethyloctadecanoate } \\
\text { Tridecyl 2,4,16-trimethylicosanoate }\end{array}$ & 0.33 & 0.12 & - & & - & \\
\hline 3557 & Hydrocarbon & 1.07 & 0.88 & 0.41 & 0.76 & 0.29 & 0.76 \\
\hline 3575 & $\begin{array}{l}\text { Tridecyl syn-2,4-dimethylhenicosanoate } \\
\text { Tetradecyl syn-2,4-dimethylicosanoate } \\
\text { Pentadecyl syn-2,4-dimethylnonadecanoate }\end{array}$ & 11.97 & 5.03 & 6.51 & 4.88 & 2.74 & 0.83 \\
\hline 3579 & $\begin{array}{l}\text { Tridecyl anti-2,4-dimethylhenicosanoate } \\
\text { Tetradecyl anti-2,4-dimethylicosanoate }\end{array}$ & 0.73 & 0.75 & 0.24 & 0.89 & - & \\
\hline
\end{tabular}


Table 1 (continued)

\begin{tabular}{|c|c|c|c|c|c|c|c|}
\hline \multirow[b]{2}{*}{$I$} & \multirow[b]{2}{*}{ Substance } & \multicolumn{2}{|c|}{ Female body } & \multicolumn{2}{|c|}{ Female web } & \multicolumn{2}{|c|}{ Male body } \\
\hline & & mean $\%$ & SD & mean $\%$ & SD & mean $\%$ & SD \\
\hline & Pentadecyl anti-2,4-dimethylnonadecanoate & & & & & & \\
\hline 3596 & Tridecyl 2,4,6-trimethylhenicosanoate & 0.17 & 0.29 & - & & - & \\
\hline 3601 & $\begin{array}{l}\text { Octadecyl 2,4,6-trimethylhexadecanoate } \\
\text { Hexadecyl 2,4,6-trimethyloctadecanoate }\end{array}$ & 2.46 & 1.32 & 1.43 & 1.23 & - & \\
\hline 3627 & Pentadecyl 2,4,16-trimethyloctadecanoate & 0.62 & 0.17 & 1.35 & 0.85 & - & \\
\hline 3656 & $\begin{array}{l}\text { 4-Methyloctadecyl 2,4-dimethylheptadecanoate } \\
\text { 4-Methylheptadecyl 2,4-dimethyloctadecanoate } \\
\text { 4-Methylhexadecyl 2,4-dimethylheptadecanoate }\end{array}$ & 0.17 & 0.16 & - & & - & \\
\hline 3670 & $\begin{array}{l}\text { Ooctadecyl 2,4-dimethylheptadecanoate } \\
\text { Heptadecyl 2,4-dimethyloctadecanoate } \\
\text { Hexadecyl 2,4-dimethylnonadecanoate } \\
\text { Pentadecyl 2,4-dimethylicosanoate } \\
\text { Tetradecyl 2,4-dimethylhenicosanoate } \\
\text { Tridecyl 2,4-dimethyldocosanoate }\end{array}$ & 2.49 & 0.96 & 1.03 & 0.87 & - & \\
\hline 3676 & Wax ester & 0.10 & 0.20 & - & & - & \\
\hline 3687 & Wax ester & $\operatorname{tr}$ & & - & & - & \\
\hline 3699 & $\begin{array}{l}\text { Nonadecyl 2,4,6-trimethylhexadecanoate } \\
\text { Octadecyl 2,4,6-trimethylheptadecanoate } \\
\text { Heptadecyl 2,4,6-trimethyloctadecanoate }\end{array}$ & 1.63 & 1.01 & 0.87 & 0.76 & - & \\
\hline 3728 & Heptadecyl 2,4,14-trimethylheptadecanoate & 0.32 & 0.18 & 0.93 & 0.81 & - & \\
\hline 3755 & Unknown & 0.43 & 0.24 & - & & - & \\
\hline 3771 & $\begin{array}{l}\text { Nonadecyl 2,4-dimethylheptadecanoate } \\
\text { Heptadecyl 2,4-dimethylnonadecanoate } \\
\text { Pentadecyl 2,4-dimethylhenicosanoate }\end{array}$ & 2.57 & 1.50 & 0.62 & 0.83 & - & \\
\hline 3802 & $\begin{array}{l}\text { Icosyl 2,4,6-trimethylhexadecanoate } \\
\text { Octadecyl 2,4,6-trimethyloctadecanoate }\end{array}$ & 5.10 & 3.28 & 2.22 & 1.73 & - & \\
\hline 3826 & $\begin{array}{l}\text { Octadecyl 2,4,14-trimethyloctadecanoate } \\
\text { Nonadecyl 2,4,14-trimethylheptadecanoate }\end{array}$ & 0.21 & 0.25 & 0.59 & 0.64 & - & \\
\hline 3855 & 4-Methylnonadecyl 2,4-dimethyloctadecanoate & 0.08 & 0.12 & - & & - & \\
\hline 3869 & $\begin{array}{l}\text { Icosyl 2,4-dimethylheptadecanoate } \\
\text { Nonadecyl 2,4-dimethyloctadecanoate } \\
\text { Octadecyl 2,4-dimethylnonadecanoate } \\
\text { Heptadecyl 2,4-dimethylicosanoate }\end{array}$ & 0.31 & 0.27 & - & & - & \\
\hline 3897 & Octadecyl 2,4,6-trimethyloctadecanoate & 0.89 & 0.70 & - & & - & \\
\hline
\end{tabular}

${ }^{1}$ The compound co-elutes with impurities and was not included in the calculation.

${ }^{2}$ Syn/anti-assignments were possible when two peaks with identical mass spectra but different $I$ occurred. The synthetic material proved that the syn-esters elute before anti-esters on the DB-5 GC phase.

unsubstituted methyl esters have a base peak of $\mathrm{m} / \mathrm{z} 74$ while the base peak is $m / z 88$ for 2-methyl-substituted methyl esters (Ryhage and Stenhagen 1960a), as is the case for a major component of both derivatized samples (Fig. 5a). Ryhage and Stenhagen (1960b) showed that polymethyl substituted methyl esters display characteristic ratios of fragment ions. The base peak at $\mathrm{m} / \mathrm{z} 88$ together with higher intensities of $\mathrm{m} / \mathrm{z} 101$ and 129 as well as the ester specific loss of C-2/C-3 + H $\left(\mathrm{m} / z\right.$ 269, $\left.[\mathrm{M}-43]^{+}\right)$and C-2/C$3 / \mathrm{C}-4+\mathrm{H}\left(\mathrm{m} / \mathrm{z} 241,[\mathrm{M}-71]^{+}\right)$indicate a 2,4-dimethyl substitution pattern (Ryhage and Stenhagen 1960b). However, additional methyl groups along the chain cannot be reliably deduced from the methyl ester spectra. Therefore, the methyl esters were transformed into 3-pyridinylmethyl esters. Such esters display a regular fragmentation pattern of successive losses of $\mathrm{CH}_{2}$ units, with branches resulting in gaps in the pattern and increased intensity in the fragments next to the branching position because of the stability of the resulting secondary radical (Harvey 1991).

The mass spectrum of this derivative is shown in Fig. 5b. The ion $\mathrm{m} / \mathrm{z} 165$ together with the base peak $\mathrm{m} / \mathrm{z} 178$ and the gap in the regular fragmentation pattern between $\mathrm{m} / \mathrm{z}$ 
Fig. 2 Total ion chromatograms of the combined cuticular extracts from male (A) and female (B) A. bruennichi. *Octadecane as internal standard for quantification
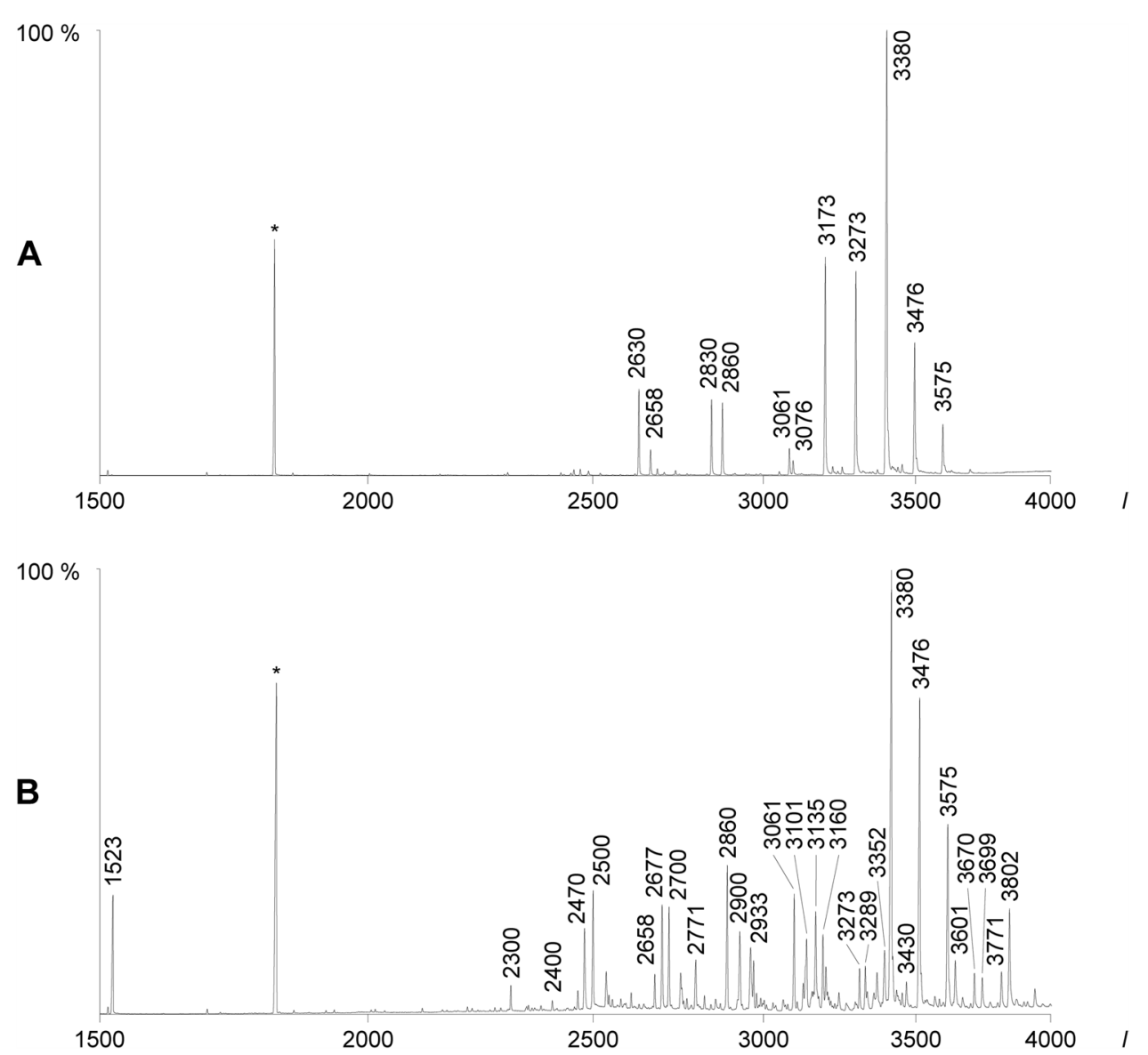

178 and $\mathrm{m} / \mathrm{z} 206$ support a 2,4-dimethylheptadecanoate structure of the acid part of the natural esters. This identification was verified using synthetic 2,4-dimethylheptadecanoic acid (9) (see below). Esterification of 9 under the same conditions as performed with the natural samples led to methyl 2,4-dimethylheptadecanoate and 3-pyridinylmethyl 2,4-dimethylheptadecanoate and confirmed our identification (mass spectra see SI, Figs. S1 and S2).

Most of the naturally occurring esters were identified using the described procedures. As can be seen from Table 1, the 2,4-dimethyl motif dominates within the natural esters. In addition, small amounts of 2,4,6-trimethylalkanoate esters and several other 2,4,x-trimethylalkanoates esters were identified using the approach described.

The alcohol parts of the esters were identified as $n$-alkan1-ols using 3-pyridinecarboxylate derivatives (nicotinates), that display a regular fragmentation pattern of successive losses of $\mathrm{CH}_{2}$ units. In addition, the nicotinates obtained from the females showed small amounts of 4-methyl alcohols. A typical mass spectrum is shown in Fig. 5c, displaying the branch-indicating gap between $\mathrm{m} / z, 164$ and 192 .

With these results in hand, we were able to identify most of the natural wax esters (Table 1). Cuticular and silk extracts from females contained acids with 17-24 carbons, with mostly 2,4-dimethyl substitution, as well as smaller amounts of 2,4,6-trimethyl substitution and various other 2,4,x-trimethyl substitution patterns. The alcohol portions were mainly $n$-alkan-1-ols, along with small amounts of 4-methyl alcohols, with chain lengths between 13-24 carbons. Extracts from males contained the same acids, although the shorter acids were more prominent. The alcohols were identified as $n$-alkan-1-ols with chain lengths between $12-15$ carbons. The identification of $n$-alkyl 2,4-dimethylalkanoates was verified through the synthesis of tetradecyl 2,4-dimethylheptadecanoate (10), as described in the following section.

The characterization of the acid and alcohol components was used to assign proposed structures to the wax esters of the natural samples basing on their mass spectra and $I$ (Table 1). The largest signals in the derivatized, as well as the natural samples, were from 2,4-dimethylalkanoates, indicating these to be the major lipid components. The remaining wax esters were assumed to have similar relative retention indices as the derivatives (Schulz 2001) and were identified as the respective $2,4, \mathrm{x}$-trimethylalkanoates. In the samples from males, 
Fig. 3 Discriminant analyses of cuticular extracts from males and females, as well as web silk from females, based on all peaks $>1 \%$ (A), only hydrocarbons (B), and only wax esters (C). Values in parentheses behind discriminant functions give percentages of explained variance
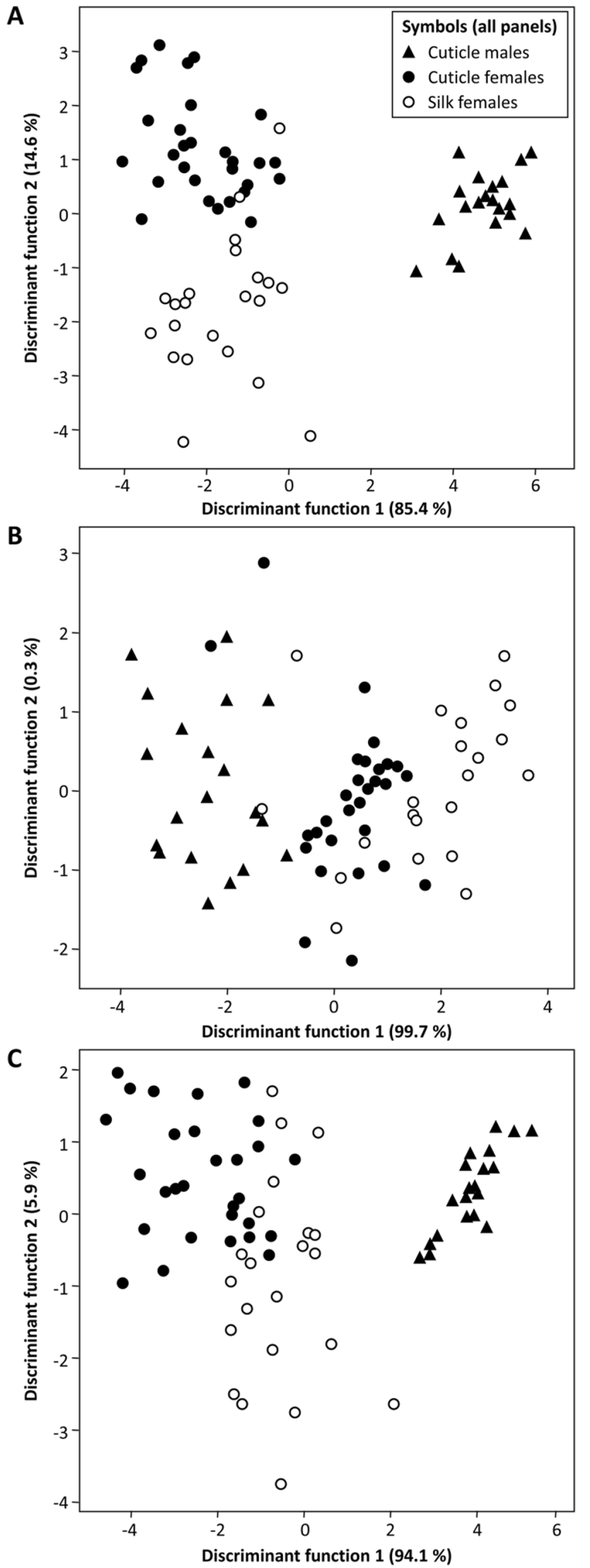
Fig. 4 Mass spectrum and structures of 2,4-dimethylalkyl wax ester mixture with $I 3273$

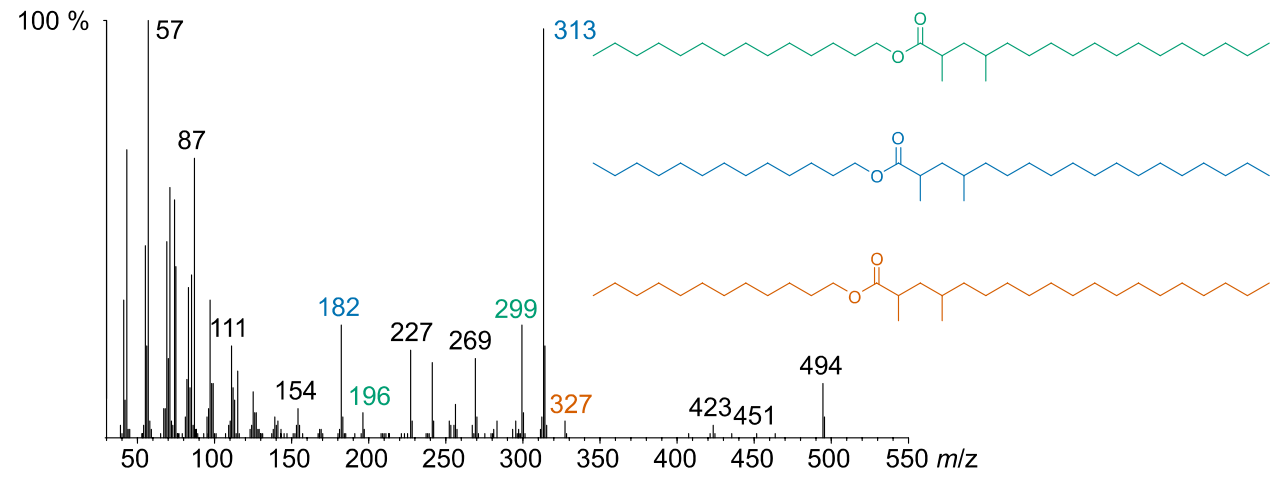

small amounts of unbranched esters were present, while in samples from females, small amounts of 4-methylalkyl 2,4-dimethylalkanoates were found.

\section{Synthesis of a Model Wax Ester}

To confirm our identification, a representative ester, tetradecyl $(2 S, 4 S)$-2,4-dimethylheptadecanoate (10) was synthesized using the strategy of Feringa et al. for the stereoselective construction of bishomolog methyl-branched acids (Fig. 6) (Des Mazery et al. 2005; Horst et al. 2007; Ruiz et al. 2007). The synthetic material was also used to determine the configuration of the natural esters. The synthesis uses an enantioselective conjugate addition of methylmagnesium bromide to $\alpha, \beta$-unsaturated thioesters, using the enantiomers of Josiphos (11) as ligands in the key steps. The configuration of each stereogenic center can thus be controlled (Horst et al. 2007).

Thus, the first stereogenic center was introduced into building block 1 with an $S / R$ ratio of 97:3 (see SI, Fig. S5) by the conjugate addition. Reduction and elongation led to compound $\mathbf{3}$, into which the second stereogenic center was introduced similarly to form compound $\mathbf{4}$ with an $R / S$ ratio of 93:7 (calculated back from the final product). Reduction of the esters to alcohol 5 and conventional Grignard-elongation delivered compound $\mathbf{7}$ that, after deprotection and oxidation, gave $(2 S, 4 S)$-acid 9 as the major product, which after esterification with 1-tetradecanol furnished ester 10 (Fig. 6).

Usually, multiple syntheses of different stereoisomers are required for elucidation of the absolute configuration of compounds with multiple stereogenic centers (see e. g. Schulz et al. 2004). We tried to solve this problem here with only one stereoselective synthesis, taking advantage of the controlled stepwise introduction of the stereogenic centers as explained in the next section.

\section{Stereochemistry of Wax Esters}

GC showed two separated peaks in the final product $\mathbf{1 0}$ in a diastereomeric ratio of 91:9 (Fig. S3). The major, first eluting peak was the $s y n$-diastereomer $(2 S, 4 S / 2 R, 4 R)$, while the smaller, second one was the anti-diasteromer $(2 S, 4 R / 2 R, 4 S)$. The methyl ester of 9 showed similar separation. The stereoisomeric mixture of the methyl esters of $\mathbf{9}$ was then separated on a chiral Hydrodex $\beta-6$ TBDM phase (Fig. 7). Peak assignment was performed using the enantiomeric excess (ee) data from the stereoselective addition. Therefore, the largest peak was the $(2 S, 4 S)$-enantiomer $(0.93 \times 0.97=0.9021$ relative peak area, ee $99.8 \%)$, while the smallest had a $(2 R, 4 R)$-configuration $(0.07 \times 0.03=0.0021)$, likely not detectable due to its low abundance. The $(2 R, 4 S)$-enantiomer $(0.07 \times 0.97=0.0679$, ee $41.8 \%$ ) was slightly more concentrated than the $(2 S, 4 R)$ enantiomer $(0.93 \times 0.03=0.0279)$. The ee data nicely showed that the consecutive introduction of chiral centers leads to a syn-product of very high ee due to the inherent ee amplification, while it is the opposite for the anti-diastereomer. The anti-enantiomers are well separated, while resolution of the syn-enantiomers was not possible (see Fig. S7). Comparison with the transesterified natural extracts revealed the natural anti-diastereomer to have the $(2 S, 4 R)$-configuration. This indicates that the natural syn-diastereomer can be assigned the $(2 R, 4 R)$-stereochemistry because the $(4 R)$-stereochemistry is fixed during biosynthesis, in contrast to the configuration at $\mathrm{C}-2$. For a detailed discussion see the Supporting Information. The formation of the anti-diastereomer might be explained by partial epimerization of $\mathrm{C}-2$ during biosynthesis of the esters. Epimerization during transesterification with TMSH is unlikely because of the reaction mechanism and the occurrence of diastereomers of some esters even in the original samples (Table 1).

In summary, over 180 cuticular compounds were detected and most of them were identified using the methods described above. While the number of hydrocarbons (72) and wax esters (75) was almost equal, the esters dominated in cuticular extracts of both females (ratio amounts esters/ hydrocarbons 64:28) and males (68:13), while both compound classes occurred in equal amounts in webs of females (44:41). 
Fig. 5 Mass spectra of methyl 2,4-dimethylheptadecanoate (a), 3-pyridinylmethyl 2,4-dimethylheptadecanoate (b), and 4-methylheptadecyl nicotinate (c), derived from wax esters a

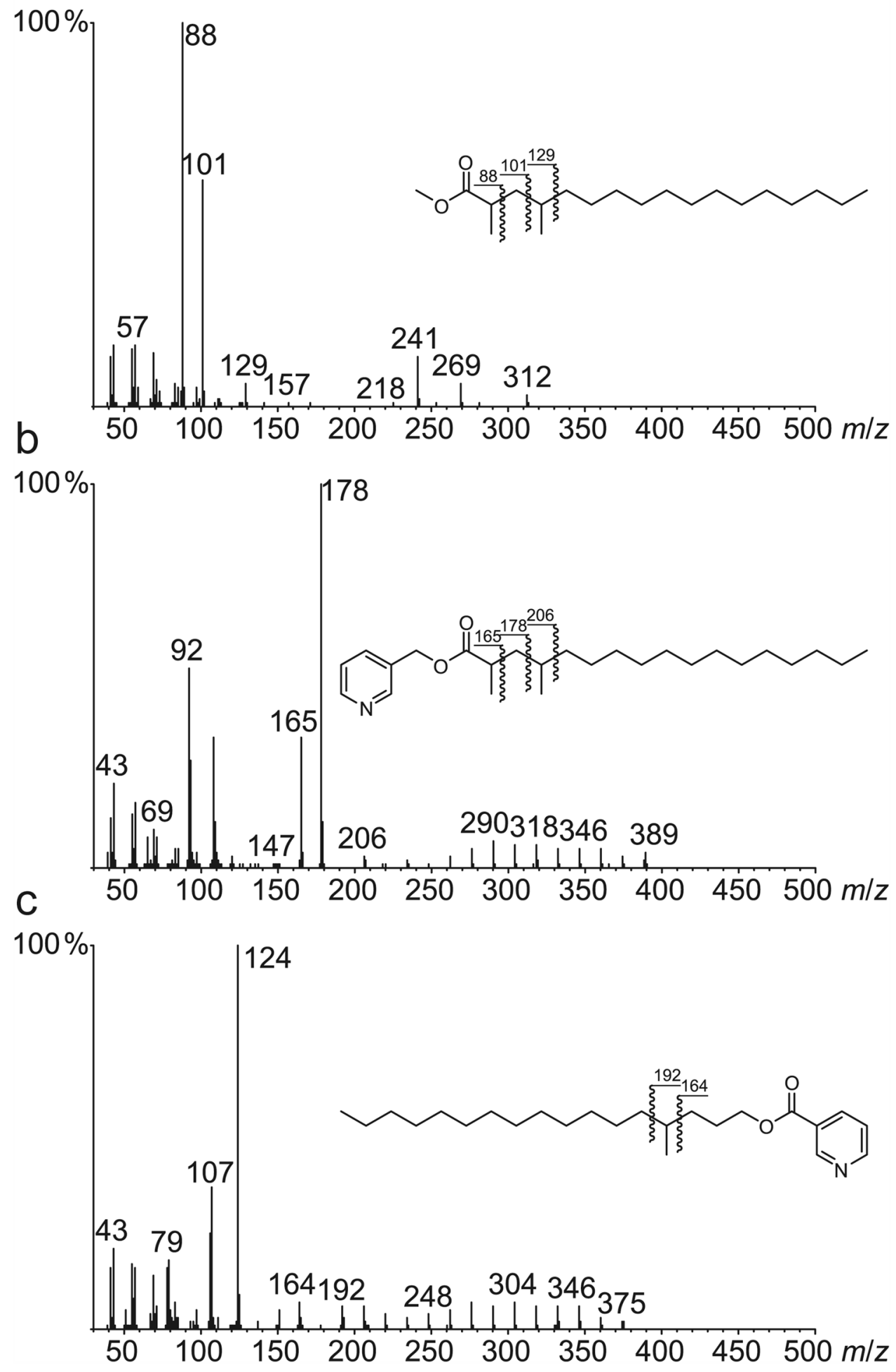

\section{Discussion}

In this study, we identified for the first time wax esters with a bishomomethyl-branched acid head group that constitute the major portion of lipids on the cuticle of both sexes and web silk of females of A. bruennichi. These cuticular compounds likely play a role in kin recognition, because family differences were observed within the wax esters, while cuticular hydrocarbons showed lower variation (Weiss and Schneider 2021). We developed an analytical procedure that 

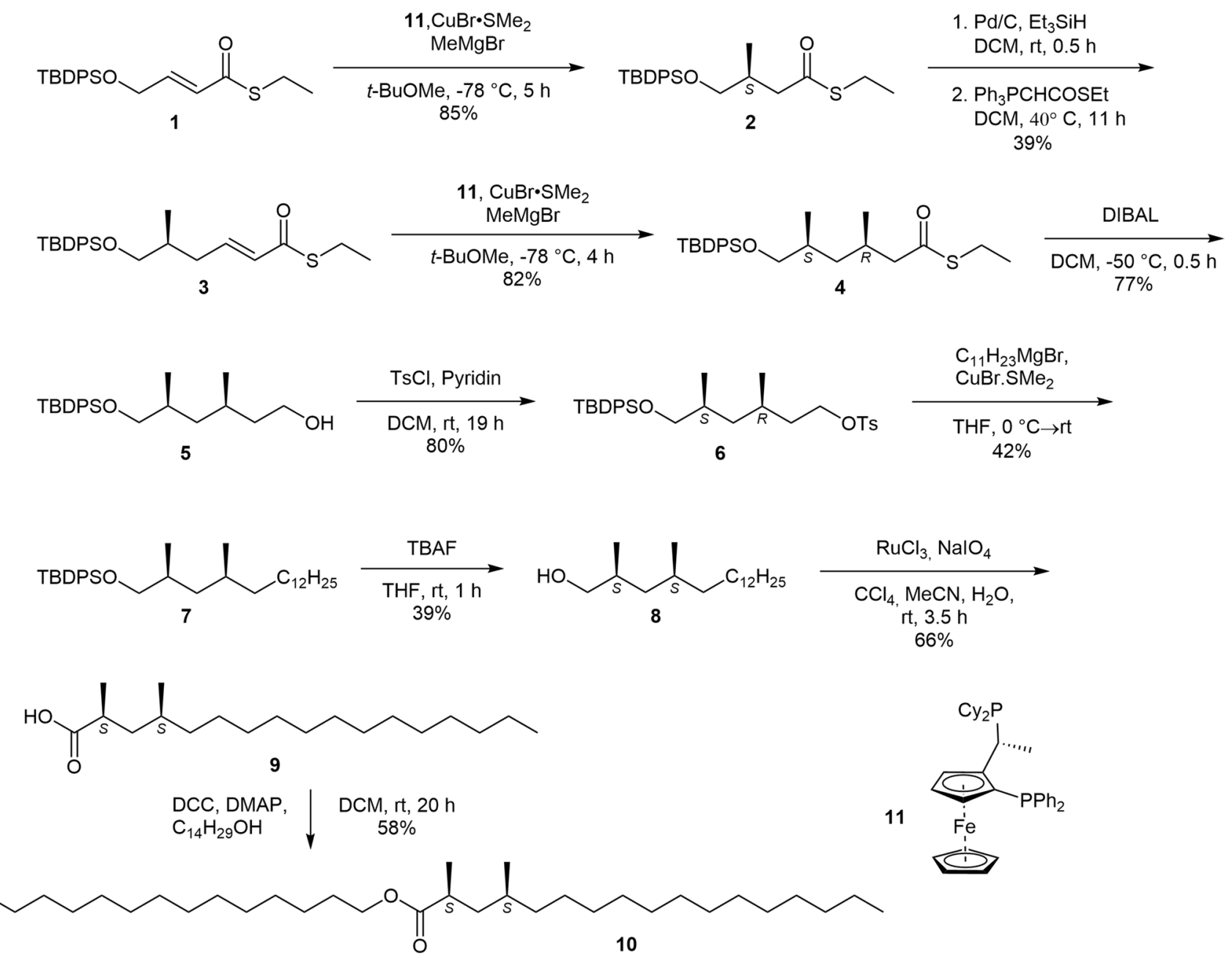

Fig. 6 Synthesis of tetradecyl $(2 S, 4 S)$-2,4-dimethylheptadecanoate (10). Please note that the configurational prefixes change during the synthesis due to the CIP-rules

can reliably be used to analyze and characterize these esters, based on previous work (Chinta et al. 2016). Furthermore, we established a synthetic procedure that allows access to the enantiomers of each ester and enables determination of the absolute configuration of the esters.

Wax esters have previously been described as cuticular compounds of spiders and scorpions (Trabalon and Bagnères 2010). Propyl esters of long-chain multiply methylbranched acids occur in complex mixtures on Anelosimus eximus (Bagnères et al. 1997), while a few shorter, sexspecific esters such as 2,8-dimethylundecyl 2,8-dimethylundecanoate or 14-methylheptadecyl 4-methylheptanoate dominate the cuticular wax of Argyrodes elevatus (Chinta et al. 2016). In contrast, the bishomomethyl-branched esters reported here represent a unique group of compounds that have not been reported from other arthropods. Nevertheless, they do not seem to be specific to Argiope, because we have also detected such compounds in the spider Pholcus phalangoides (S. Schulz, unpublished).

The structures of the hydrocarbons identified here are not very different from those found in other spiders. An enhanced concentration of 2-methyl-branched alkanes with an even number of carbons was observed, in line with reports from other spider species (Schulz 2001, 2013), a trait usually not observed within insect hydrocarbons. A close similarity in compound structures between samples from females and males is evident, but sex differences exist in the number of compounds, which is lower in males, and the relative proportions of the components. Consequently, cuticular profiles of males and females were clearly separated by discriminant analyses based on relative peak areas, while the profile of silk from females more closely resembled cuticular extracts from females. Specifically, females showed a larger number of trimethyl-substituted esters and a slightly higher 


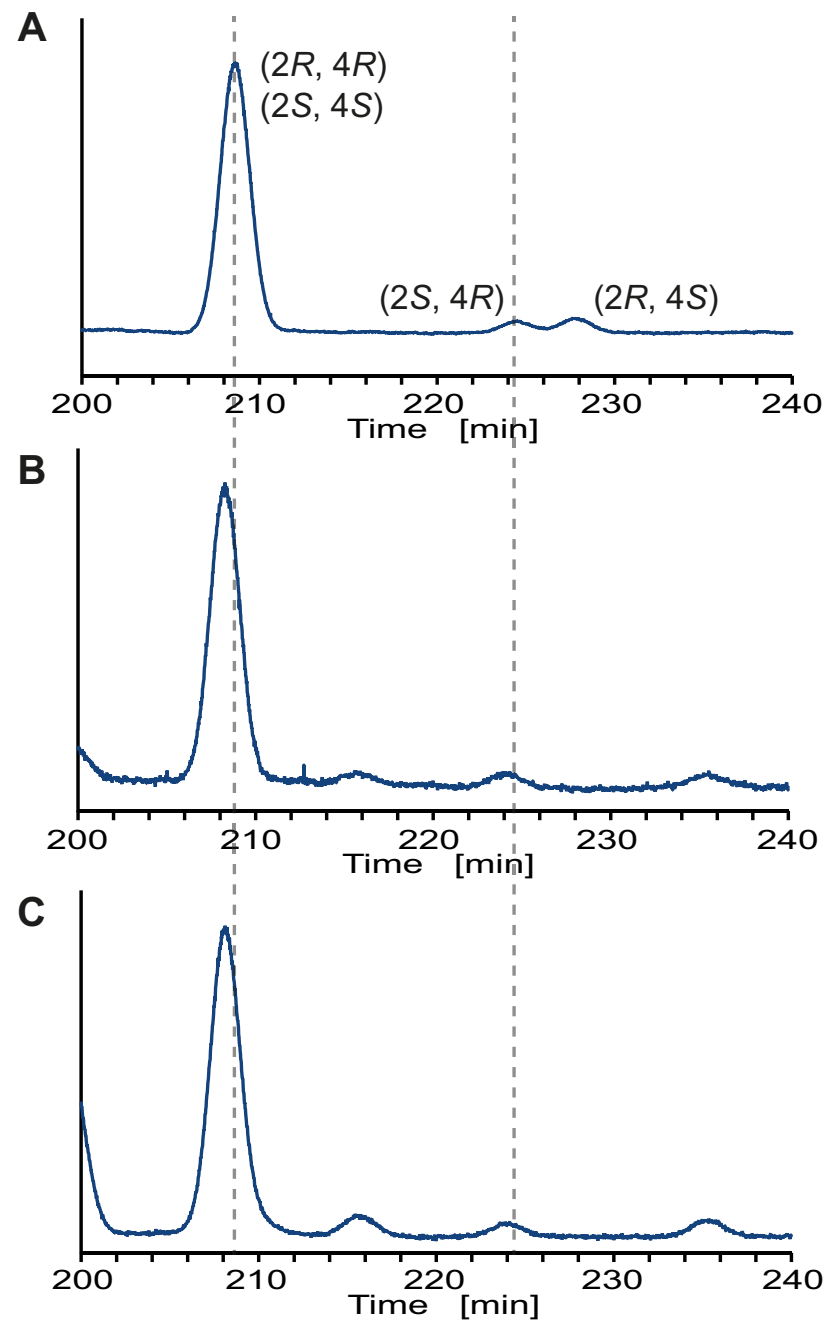

Fig. 7 Resolution of the methyl ester of 2,4-dimethylheptadecanoic acid (9) on a chiral GC phase. Separation was performed using a Hydrodex $\beta$-6TBDM phase $\left(30.0 \mathrm{~m} \times 0.25 \mathrm{~mm}, 1.5 \mathrm{~mL} / \mathrm{min} \mathrm{H}_{2}\right.$, initial temp. $50{ }^{\circ} \mathrm{C}$ then $10{ }^{\circ} \mathrm{C} \min ^{-1}$ to $125{ }^{\circ} \mathrm{C}$ holding time for $240 \mathrm{~min}$, then with $10{ }^{\circ} \mathrm{C} \min ^{-1}$ to final temp. $230{ }^{\circ} \mathrm{C}$ ). A: methyl ester of synthetic $(2 S, 4 S)$-2,4-dimethylheptadecanoic acid (9). B: methyl ester of the transesterified sample of a body extract of male Argiope bruenichi. C: same with female A. bruenichi

molecular weight, ranging from 480 to $564 \mathrm{amu}$, while those in males ranged from 466 to $536 \mathrm{amu}$. Both sexes share tridecyl syn-2,4-dimethylnonadecanoate (522 amu) as a major component. However, females also have considerable amounts of tridecyl syn-2,4-dimethylicosanoate and tridecyl syn-2,4-dimethylhenicosanoate (536 amu), whereas males possess more tridecyl 2,4-dimethyloctadecanoate (494 amu) and tridecyl syn-2,4-dimethylheptadecanoate (508 amu). Hence females are characterized by larger esters than males, but we also found male specific wax esters, namely tridecyl and tetradecyl esters of octadecanoic and several 2,4,14-trimethylalkanoic acids.
In summary, we have clarified the complex cuticular chemistry of A. bruennichi. With these data and synthetic material, behavioral assays can be planned to elucidate which signals are used for kin recognition. Although synthesis of a complete array of esters seems out of reach, addition of individual esters to manipulate cuticular chemistry might be a promising approach for further research.

Supplementary Information The online version contains supplementary material available at https://doi.org/10.1007/s10886-021-01338-y .

Acknowledgements We thank the Deutsche Forschungsgemeinschaft (DFG) for grants to J. M. S. (SCHN 561/15-1) and K. W. (WE 6634/1-1).

Funding Open Access funding enabled and organized by Projekt DEAL. This work was funded by the Deutsche Forschungsgemeinschaft (DFG), through grants to J. M. S. (SCHN 561/15-1) and K. W. (WE 6634/1-1).

Data Availability Not applicable.

Code Availability Not applicable.

\section{Declarations}

Conflicts of Interest The authors have no financial or proprietary interests in any material discussed in this article.

Open Access This article is licensed under a Creative Commons Attribution 4.0 International License, which permits use, sharing, adaptation, distribution and reproduction in any medium or format, as long as you give appropriate credit to the original author(s) and the source, provide a link to the Creative Commons licence, and indicate if changes were made. The images or other third party material in this article are included in the article's Creative Commons licence, unless indicated otherwise in a credit line to the material. If material is not included in the article's Creative Commons licence and your intended use is not permitted by statutory regulation or exceeds the permitted use, you will need to obtain permission directly from the copyright holder. To view a copy of this licence, visit http://creativecommons.org/licenses/by/4.0/.

\section{References}

Adams SA, Gerbaulet M, Schulz S, Gillespie RG, Uhl G (2021) Chemical species recognition in a Tetragnatha Spider (Araneae: Tetragnathidae). J Chem Ecol 37:4763-4772. https://doi.org/10.1007/ s10886-020-01237-8

Aitchison J (2003) The statistical analysis of compositional data. Blackburn Press, Caldwell

Bagnères A-G, Trabalon M, Blomquist GJ, Schulz S (1997) Waxes of the Social Spider Anelosimus eximus; Abundance of Novel n-Propyl Esters of Long-Chain Methyl-Branched Fatty Acids. Arch Insect Biochem Physiol 36:295-314

von Beeren C, Hashim R, Witte V (2012) The social integration of a Myrmecophilous Spider does not depend exclusively on chemical mimicry. J Chem Ecol 38:262-271. https://doi.org/10.1007/ s10886-012-0083-0 
Blomquist GJ, Bagnères A-G (2010) Insect Hydrocarbons: Biology, biochemistry, and chemical ecology. Cambridge University Press, Cambridge

Carlson DA, Bernier UR, Sutton BD (1998) Elution Patterns from Capillary GC for Methyl-Branched Alkanes. J Chem Ecol 24:1845-1866

Chinta SP, Goller S, Lux J, Funke S, Uhl G, Schulz S (2010) The sex pheromone of the wasp spider Argiope bruennichi. Angew Chem Int Ed 49:2033-2036

Chinta SP, Goller S, Uhl G, Schulz S (2016) Identification and Synthesis of Branched Wax-type Esters, Novel Surface Lipids from the Spider Argyrodes elevatus (Araneae: Theridiidae). Chem Biodivers 13:1202-1220. https://doi.org/10.1002/cbdv.20160 0020

Cory A-L, Schneider JM (2016) Old maids have more appeal: effects of age and pheromone source on mate attraction in an orb-web spider. PeerJ 4:e1877. https://doi.org/10.7717/peerj.1877

Des Mazery R, Pullez M, López F, Harutyunyan SR, Minnaard AJ, Feringa BL (2005) An iterative catalytic route to enantiopure deoxypropionate subunits: asymmetric conjugate addition of grignard reagents to $\alpha, \beta$-unsaturated thioesters. J Am Chem Soc 127:9966-9967. https://doi.org/10.1021/ja053020f

El-Sayed A (2019) The Pherobase. The Pherobase. http://www.phero base.com/. Accessed 6/26/2019

Fischer A (2019) Chemical communication in spiders - a methodological review. J Arachnol 47:1. https://doi.org/10.1636/0161-820247.1.1

Foelix RF (2011) Biology of spiders, 3rd edn. Oxford Univ. Press, Oxford

Gaskett AC (2007) Spider sex pheromones: emission, reception, structures, and functions. Biol Rev 82:26-48

Greenfield MD (2002) Signalers and receivers. Oxford University Press, Oxford New York

Grinsted L, Bilde T, D'Ettorre P (2011) Cuticular hydrocarbons as potential kin recognition cues in a subsocial spider. Behav Ecol 22:1187-1194. https://doi.org/10.1093/beheco/arr105

Harvey DJ (1982) Picolinyl esters as derivatives for the structural determination of long chain branched and unsaturated fatty acids. Biomed Mass Spectrom 9:33-38

Harvey DJ (1991) Lipids from the Guinea Pig Harderian Gland: Use of picolinyl and other pyridine-containing derivatives to investigate the structures of novel branched-chain fatty acids and glycerol ethers. Biol Mass Spectrom 20:61-69

Howard RW, Blomquist GJ (2005) Ecological, behavioral, and biochemical aspects of insect hydrocarbons. Annu Rev Entomol 50:371-393. https://doi.org/10.1146/annurev.ento.50.071803. 130359

ter Horst B, Feringa BL, Minnaard AJ (2007) Catalytic Asymmetric Synthesis of Phthioceranic Acid, a Heptamethyl-Branched Acid from Mycobacterium tuberculosis. Org Lett 9:3013-3015. https:// doi.org/10.1021/o1071078o

McLafferty FW, Turecek F (1993) Interpretation of Mass Spectra, 4th edn. University Science Books, Mill Valley

Müller K-D, Husmann H, Nalik HP, Schomburg G (1990) Trans-esterification of fatty acids from microorganisms and human blood serum by trimethylsulfonium hydroxide (TMSH) for GC analysis. Chromatographia 30:245-248

Nessler SH, Uhl G, Schneider JM (2007) Genital damage in the orbweb spider Argiope bruennichi (Araneae: Araneidae) increases paternity success. Behav Ecol 18:174-181. https://doi.org/10. 1093/beheco/arl074

NIST 08 mass spectral library (2008) NIST 08 mass spectral library. Wiley and Sons, Hoboken
Prouvost O, Trabalon M, Papke M, Schulz S (1999) Contact sex signals on web and cuticle of Tegenaria atrica (Araneae, Agenelidae). Arch Insect Biochem Physiol 40:194-202

Ruhland F, Schulz S, Hervé MR, Trabalon M (2019) Do wolf spiders' egg-sacs emit tactochemical signals perceived by mothers? Behav Ecol 30:570-581. https://doi.org/10.1093/beheco/ ary 197

Ruiz BM, Geurts K, Fernández-Ibáñez MA, ter Horst B, Minnaard AJ, Feringa BL (2007) Highly versatile enantioselective conjugate addition of Grignard reagents to $\alpha, \beta$-unsaturated thioesters. Org Lett 9:5123-5126. https://doi.org/10.1021/ol702425a

Ryhage R, Stenhagen E (1960a) Mass spectrometric studies: IV. Esters of monomethyl-substituted long chain carboxylic acids. Ark Kemi 15:291-304

Ryhage R, Stenhagen E (1960b) Mass spectrometric studies: V. Methyl esters of monoalkyl-substituted acids with ethyl or longer side chain and methyl esters of di- and polyalkyl-substituted acids. Ark Kemi 15:333-351

Schulz S (2001) Composition of the silk lipids of the spider Nephila clavipes. Lipids 36:637-647

Schulz S (2004) Semiochemistry of spiders. In: Cardé RT, Millar JG (eds) Advances in Chemical Ecology. Cambridge University Press, Cambridge, pp 110-150

Schulz S (2013) Spider Pheromones - a Structural Perspective. J Chem Ecol 39:1-14. https://doi.org/10.1007/s10886-012-0231-6

Schulz S, Fuhlendorff J, Reichenbach H (2004) Identification and synthesis of volatiles released by the myxobacterium Chondromyces crocatus. Tetrahedron 60:3863-3872

Schulz S, Toft S (1993) Branched long chain alkyl methyl ethers: a new class of lipids from spider silk. Tetrahedron 49:6805-6820. https://doi.org/10.1016/S0040-4020(01)80424-5

Trabalon M (2011) Agonistic interactions, cuticular and hemolymphatic lipid variations during the foraging period in spider females Brachypelma albopilosa (Theraphosidae). J Insect Physiol 57:735-743

Trabalon M, Assi-Bessekon D (2008) Effects of web chemical signatures on intraspecific recognition in a subsocial spider, Coelotes terrestris (Araneae). Anim Behav 76:1571-1578

Trabalon M, Bagnères A-G (2010) Contact recognition pheromones in spiders and scorpions. In: Blomquist GJ, Bagnères A-G (eds) Insect Hydrocarbons: Biology, biochemistry, and chemical ecology. Cambridge University Press, Cambridge, UK, New York, pp 344-374

Trabalon M, Bagnères A-G, Hartmann N, Vallet MA (1996) Changes in Cuticular Compounds Compounds Composition During the Gregarious Period and After Dispersal of the Young in Tegenaria atrica (Araneae, Agelenidae). Insect Biochem Molec Biol 26:77-84. https://doi.org/10.1016/S0965-1748(98) 00066-6

Trabalon M, Bagnères A-G, Roland C (1997) Contact Sex Signals in Two Sympatric Spider Species, Tegenaria domestica and Tegenaria pagana. J Chem Ecol 23:747-758

Uhl G, Nessler SH, Schneider J (2007) Copulatory mechanism in a sexually cannibalistic spider with genital mutilation (Araneae: Araneidae: Argiope bruennichi). Zoology 110:398-408. https:// doi.org/10.1016/j.zool.2007.07.003

Uhl G, Nessler SH, Schneider JM (2010) Securing paternity in spiders? A review on occurrence and effects of mating plugs and male genital mutilation. Genetica 138:75-104. https://doi.org/10.1007/ s10709-009-9388-5

van den Dool H, Kratz P (1963) A generalization of the retention index system including linear temperature programmed gas-liquid partition chromatography. J Chromatogr 11:463-471

Vetter W, Meister W (1981) Nicotinates as derivatives for the mass spectrometric investigation of long chain alcohols. Org Mass Spectrom 16:118-122 
Weiss K, Schneider JM (2021) Family-specific chemical profiles provide potential kin recognition cues in the sexually cannibalistic spider Argiope bruennichi. Biol Lett 17:202110260. https://doi. org/10.1098/rsbl.2021.0260

Welke KW, Schneider JM (2010) Males of the orb-web spider Argiope bruennichi sacrifice themselves to unrelated females. Biol Lett 6:585-588. https://doi.org/10.1098/rsbl.2010.0214

Welke KW, Zimmer SM, Schneider JM (2012) Conditional monogyny: female quality predicts male faithfulness. Front Zool 9:7. https:// doi.org/10.1186/1742-9994-9-7
Witte V, Foitzik S, Hashim R, Maschwitz U, Schulz S (2009) Fine tuning of social integration by two myrmecophiles of the ponerine army ant, Leptogenys distinguenda. J Chem Ecol 35:355-367

Xiao Y-H, Zhang J-X, Li S-Q (2010) Male-specific (Z)-9-tricosene stimulates female mating behaviour in the spider Pholcus beijingensis. Proc R Soc B Biol Sci 277:3009-3018 